\title{
Intra-epithelial non-canonical Activin A signalling safeguards prostate progenitor quiescence
}

3 Francesco Cambuli ${ }^{1,3, \#, *, \delta}$, Veronica Foletto ${ }^{1, \#}$, Alessandro Alaimo ${ }^{1}$, Dario De Felice ${ }^{1}$, Francesco

4 Gandolfi $^{2}$, Maria Dilia Palumbieri ${ }^{1}$, Michela Zaffagni ${ }^{1}$, Sacha Genovesi ${ }^{1}$, Marco Lorenzoni ${ }^{1}$, Martina

5 Celotti ${ }^{1}$, Emiliana Bertossio ${ }^{1}$, Giosuè Mazzero ${ }^{4}$, Arianna Bertossi ${ }^{1}$, Alessandra Bisio ${ }^{1}$, Francesco

Berardinelli $^{5,6}$, Antonio Antoccia ${ }^{5}$, Marco Gaspari ${ }^{7}$, Mattia Barbareschi ${ }^{4}$, Michelangelo Fiorentino ${ }^{8}$,

Michael M. Shen ${ }^{3}$, Massimo Loda ${ }^{9}$, Alessandro Romanel ${ }^{2}$, and Andrea Lunardi ${ }^{1, *}$.

${ }^{1}$ The Armenise-Harvard Laboratory of Cancer Biology \& Genetics, Department of Cellular, Computational and Integrative Biology (CIBIO), University of Trento, 38123 Trento (TN), Italy.

$10{ }^{2}$ Laboratory of Bioinformatics and Computational Genomics, Department of Cellular, Computational and 11 Integrative Biology (CIBIO), University of Trento, 38123 Trento (TN), Italy.

$12{ }^{3}$ Department of Medicine, Genetics and Development, Urology, Systems Biology, Herbert Irving 13 Comprehensive Cancer Center, Columbia University Irving Medical Center, New York, NY 10032, USA.

$14{ }^{4}$ Santa Chiara Hospital-APSS, Trento, 38122 Trento (TN), Italy.

$15{ }^{5}$ Department of Science, University of Roma Tre, 00146 Roma, Italy.

$16{ }^{6}$ Laboratory of Neurodevelopment, Neurogenetics and Molecular Neurobiology Unit, IRCCS Santa Lucia 17 Foundation, 00143, Rome, Italy

$18{ }^{7}$ Department of Experimental and Clinical Medicine, University of Catanzaro, 88100 Catanzaro (CZ), Italy.

$19{ }^{8}$ Department of Experimental, Diagnostic and Specialty Medicine, University of Bologna, 40138 Bologna 20 (BO), Italy.

$21{ }^{9}$ Department of Pathology and Laboratory Medicine, Weill Medical College of Cornell University, New York, 22 NY10021, USA.

$23 \quad$ \# These authors contributed equally as first authors

24 * Corresponding author: +1 6468883997 cambulif@mskcc.org

$25 *$ Corresponding author: +39 0461285288 andrea.lunardi@unitn.it

$26{ }^{\delta}$ Present address: Molecular Pharmacology Program, Sloan Kettering Institute, Memorial Sloan Kettering 27 Cancer Center, New York, NY 10065, USA

\section{Keywords}




\section{Abstract}

The healthy prostate is a relatively quiescent tissue. Yet, prostate epithelium overgrowth is a common condition during ageing, associated with urinary dysfunction and tumorigenesis. For over thirty years, TGF- $\beta$ ligands have been known to induce cytostasis in a large variety of epithelia, but the intracellular pathway mediating this signal in the prostate, as well as its relevance for quiescence, have remained elusive.

Here, using mouse prostate organoids to model epithelial progenitors, we found that intraepithelial non-canonical Activin A signalling inhibited cell proliferation in a Smad-independent manner. Mechanistically, Activin A triggered Tak1 and p38 MAPK activity, leading to p16 and p21 nuclear import. Spontaneous evasion from this quiescent state occurred upon prolonged culture, due to reduced Activin A secretion, a condition associated with DNA replication stress and aneuploidy. Organoids capable to escape quiescence in vitro were also able to implant with increased frequency into immunocompetent mice.

Our study demonstrates that non-canonical Activin A signalling safeguards epithelial quiescence in the healthy prostate, with potential implications for the understanding of cancer initiation, and the development of therapies targeting quiescent tumour progenitors. 


\section{Introduction}

The healthy prostate is a relatively quiescent tissue during adulthood ${ }^{1,2}$. In contrast, the overgrowth of the prostatic epithelium is one of the most common conditions experienced by ageing men, being linked with urinary dysfunction and tumorigenesis ${ }^{3}$. The molecular mechanisms causing exit from quiescence are poorly understood. Chronic inflammation - potentially induced by infection (e.g., prostatitis $)^{4-6}$, chemical damage (e.g., urine reflux $)^{7}$, physical trauma (e.g., corpora amylacea $)^{8}$, dietary carcinogens ${ }^{9}$, obesity ${ }^{10}$, hormonal imbalance (e.g., low systemic androgen levels) $)^{11,12}$, and ageing $^{13}$ - has been implicated in DNA damage, oxidative stress, and atrophy, leading to a proliferative response ${ }^{14,15}$. Considering the high frequency of these events, it would be logical to hypothesize specialized mechanisms to safeguard epithelial quiescence, but they have been rarely investigated.

It has long been known that Transforming Growth Factor $\beta$ (TGF- $\beta$ ) signalling inhibits the proliferation of a large variety of epithelial cell types ${ }^{16,17}$, including those of the prostate ${ }^{18}$. SMAD factors are the canonical intracellular mediators of this signalling, but additional non-canonical pathways can also be triggered by TGF- $\beta$ receptors ${ }^{19,20}$. In gastrointestinal (GI) carcinomas (e.g., pancreas, colon), the canonical pathway is frequently mutated ${ }^{21,22}$. However, outside of the GI tract, TGF- $\beta /$ SMAD components are rarely inactivated in tumours, leaving unexplained the nature of the intracellular signalling responsible for the cytostatic effect of TGF- $\beta^{23,24}$.

Enhanced Tgf- $\beta$ signalling has been linked with the presence of quiescent epithelial progenitors in the proximal/periurethral region of the mouse prostate ${ }^{25,26}$. Recent single-cell studies have confirmed the enrichment of a variety of epithelial progenitors - basal, luminal proximal (LumP), and periurethral ( $\mathrm{PrU}$ ) cells - in this anatomical district, though also present at low frequency in the distal compartment ${ }^{27-32}$. Such cells are known to be particularly quiescent during homeostasis $^{33,34}$, but also to exhibit extensive regenerative potential in ex-vivo assays ${ }^{31,35}$.

Thus, the TGF- $\beta$ induced cytostatic response in epithelial progenitors may be relevant for the control of quiescence, but the complexity of this pathway, the lack of interpretable genetic alterations in patients, and the heterogeneous cellular composition of the prostate, have so far hampered mechanistic investigations. Here, we reasoned that prostate organoid models ${ }^{36,37}$ - in combination with orthotopic transplantation approaches - may provide a biologically relevant, and experimentally amenable, system for addressing this question. 


\section{Results}

Mouse prostate organoid cultures enable the continuous expansion of epithelial progenitors in a near-physiological manner.

Initially, we set out to assess whether mouse prostate organoids are a representative and informative model for the study of the prostate epithelium, in light of recent discoveries on prostate cellular heterogeneity and dynamics ${ }^{27,28,30-32,34,38}$. Considering our interest in signalling, we focused on a culture method in defined media conditions. This protocol relies on a mix of growth factors and inhibitors, including Egf, Noggin, R-spondin 1, the Tgf- $\beta$ receptors inhibitor A83-01, and dihydrotestosterone (ENRAD) ${ }^{36}$. We generated a biobank of mouse prostate organoids, starting from bulk populations of cells from distinct prostate lobes and mouse strains (Fig. 1a, Supplementary Fig $\mathrm{S} 1)$. In line with previous studies ${ }^{36,37}$, we found that, upon tissue dissociation, only a small fraction of cells (approx. 1\%) was capable to generate organoids in culture, and that organoid-forming efficiency increased over passages, suggesting enrichment for epithelial progenitors (Fig. 1b). To gain greater insights, we longitudinally tracked organoid formation - from single cells to fully formed organoids - and we observed a progressive expansion of cells expressing the progenitor epithelial surface antigen Sca-1 (encoded by Ly6a) (Fig. 1c) ${ }^{31,35}$. Thereafter, the level of Sca-1 appeared to be stable over a long culture period (e.g., 10 weeks). To extend our observations, we performed transcriptomic analyses on three organoid lines derived from distinct mouse prostate lobes (Fig. 1d,e). Consistently with enrichment for epithelial progenitors, organoids expressed high levels of genes specific for the proximal and periurethral compartments (e.g., Psca, Tacstd2, and Ly6d), as well as basal (e.g., Krt5, Krt14, Trp63) and luminal marker genes (e.g., Krt8, Ar, Foxa1). In contrast, distal luminal markers were barely detectable (e.g., Nkx3.1, Pbsn, Sbp). Histological (H\&E) and immunofluorescent (IF) analyses confirmed that prostate organoids, for the most part, are made up of a bilayer of cuboidal cells, displaying progenitor marker proteins (e.g., Krt7, Ppplr1b), and resembling the cyto-architecture of the periurethral/proximal compartment (Fig. 1f-h). As expected for periurethral/proximal cells - which are known to be castration resistant - mouse prostate organoids were reversibly dependent on androgen for lumen formation, but not for their survival (Supplementary Fig. S2).

The epithelium of the prostate is characterized by a slow cellular turnover. In contrast, prostate organoids appeared to proliferate indefinitely - while retaining low levels of genomic instability (Supplementary Fig. S3) - raising the question of how culture conditions enable persistent cycling in a near-physiological manner. Either an excess of stimulatory cues in culture, or a lack of inhibitory 
ones - or both - may explain the shift from homeostatic quiescence in vivo to unrestrained mitotic

121 activity in vitro. Using a 'n -1 approach' for assessing the requirement for growth factors and 122 inhibitors in culture (Supplementary Fig. S4a), we found that prostate organoids are strictly dependent 123 on Egf, with sub-nanogram concentration levels being sufficient for cell cycle progression 124 (Supplementary Fig. S4b,c). Still - based on ligand/receptor expression patterns - Egf signalling 125 alone is unlikely to explain the excess of proliferation in culture. Indeed, Egf is highly transcribed by 126 distal luminal cells in the adult prostate ${ }^{28}$, and progenitor cells express the Egf receptor in vivo 127 independently of their proximal or distal location - at levels comparable to those observed in vitro 128 (Supplementary Fig. S4d). Therefore, we focused on the requirement for the Tgf- $\beta$ receptor inhibitor A83-01 for the continuous expansion of mouse prostate organoid cultures.

Intra-epithelial non-canonical Activin A signalling is a key mediator of the Tgf- $\beta$ induced cytostatic response in mouse prostate organoids.

Upon A83-01 withdrawal, organoids displayed a marked reduction in EdU incorporation within 24 hours, demonstrating a cytostatic response in this model (Fig. 2a, Supplementary Fig. S5a). A83-01 is a potent inhibitor of three type-I Tgf- $\beta$ family receptors, and two of them - Acvr1b and Tgfbr1 (also known as Alk4 and Alk5) - were found to be expressed in prostate organoids 136 (Supplementary Fig. S5b). Paracrine and autocrine ligand-receptor interactions have been demonstrated to negatively regulate epithelial proliferation ${ }^{16,39}$ leading us to investigate the release of Tgf- $\beta$ ligands in organoid cultures. We employed a click-chemistry approach to enrich for secreted proteins released in the culture medium, followed by mass-spectrometry analysis ${ }^{40}$. Over multiple experiments, we consistently recovered Activin A peptides (encoded by the Inhba gene) in the organoid supernatant, and only in one instance, a Tgfbl peptide (Fig. 2b). We were also able to immunolocalize Acvr1b in organoid cells, and in progenitor cells in vivo (Fig. 2c), with an expression pattern similar to Egfr (Supplementary Fig. S4d). Replacement of A83-01 with Follistatin - a wellknown Activin A inhibitor ${ }^{41}$ - was sufficient to sustain proliferation, revealing a prominent role for the latter in inducing a cytostatic response (Fig. 2d). To gain greater insights on the downstream pathway, we boosted Tgf- $\beta$ receptor activity by combining A83-01 withdrawal with the supplementation of distinct ligands. Activin A was found to enhance the non-canonical arm of Tgf- $\beta$ signalling - mediated by the Tgf- $\beta$ activated kinase, Tak1 (encoded by the Map3k7 gene), and the downstream p38 MAPKs - as well as the accumulation of the cell cycle inhibitor p21 (Fig. 2e). In contrast, Tgfb1 increased the activity of the canonical Tgf- $\beta$ pathway - via Smad $2 / 3$ phosphorylation, but with little, if any, alteration of p21 levels. This dichotomy prompted us to functionally test the 
role of the canonical, and non-canonical pathway, respectively. Disruption of the canonical pathway, via shRNA-mediated silencing of Smad4, did not alter the cytostatic response upon A83-01 withdrawal (Supplementary Fig. S6). In contrast, the inhibition of either Tak1 or the structurally related p38 $\alpha$ and p38 $\beta$ MAPKs - using a variety of inhibitors (Fig. 2f,g; Supplementary Fig. S7a,b) - was sufficient to ensure organoid expansion in the absence of Tgf- $\beta$ receptor blockade.

TGF- $\beta$ receptors, and cytokine-stimulated receptors (e.g., IL-1, TNF, type-I interferons), are known to converge on TAK1-p38 MAPK signalling to activate a variety of downstream factors controlling immune- and stress-related responses, including well-characterized transcriptional that in the absence of Tgf- $\beta$ receptors inhibition, Tak $1-\mathrm{p} 38 \alpha / \beta$ signalling resulted in phosphorylation and nuclear shuttling of immune-related transcription factors (e.g., Stat1/2, NF-kB), which led to the transcription of immune related gene sets (e.g., induced by TNF or type-I interferons) ${ }^{46}$, as well as to the phosphorylation of the stress-related kinase Mapkapk2 (Fig. 2h,i; Supplementary Fig. S7c-f). Importantly, Tak1-p38 MAPK activity was associated with the nuclear accumulation of the key cell cycle inhibitors p21 and p16 (Fig. 2h). induce a cytostatic response in mouse prostate organoids.

Evasion from the Tgf- $\beta$ induced cytostatic response via downregulation of intra-epithelial

\section{Activin A signalling.}

We reasoned that our biobank may offer the opportunity to discover mechanisms of evasion from the Tgf- $\beta$ induced cytostatic response, mediated by intra-epithelial Activin A signalling, in an unbiased manner. Therefore, we attempted to culture multiple prostate organoid lines in the absence of A83-01, waiting for the potential emergence of clones capable to thrive in these conditions. Out of nine prostate organoid lines (from three distinct mice), six were irreversibly lost within few weeks. Still, three lines survived for an extended period, with two of them eventually adapting to the absence of A83-01 and recovering the ability to be passaged at clonal density (Fig. 3a,b; Supplementary Fig. S8). We performed bulk-RNA sequencing on the C57\#1 DLP organoid line in the presence of A8301, one day after inhibitor withdrawal, and upon adaptation; additionally, we sequenced the C57\#3

180 DLP organoid line in control conditions and following adaptation (Fig. 3c). We focused on 181 transcriptional alterations shared by both lines displaying adaptation to A83-01 withdrawal. First, we noticed that gene signatures associated with ATR signalling were strongly upregulated upon 
183 adaptation (Fig. 3d), indicating potential DNA replication stress in S-phase, a finding consistent with

184 phosphorylation of the cell cycle Checkpoint kinase 1 (Chek1) (Fig. 3e). Of note, in the adapted 185 C57\#1 DLP line we detected widespread genomic instability and telomere doublets, a hallmark of 186 DNA replication stress, while retaining an intact p53 pathway (Fig. 3f-h; Supplementary Fig. S9). 187 Second, in both adapted organoid lines, we observed the downregulation of immune-related 188 transcriptional programmes (e.g., type-I interferon stimulated genes), suggesting an impairment of 189 non-canonical Activin A signalling (Supplementary Fig. S10). Consistently, we found that adapted 190 organoid lines significantly reduced Activin A secretion in culture (Fig. 3i), and that exogenous 191 Activin A (but not Tgfb1) was sufficient to restore the cytostatic response (Fig. 3j,k; Supplementary 192 Fig. S11).

Thus, mouse prostate organoids are capable to dampen the Tgf- $\beta$ induced cytostatic response by downregulation of intra-epithelial Activin A signalling.

Mouse prostate organoids with reduced intra-epithelial Activin A signalling display enhanced engraftment upon syngeneic transplantation.

We wondered whether a reduced intra-epithelial Activin A signalling may release the progenitor proliferative potential in response to basal growth stimuli, within the relative quiescent microenvironment of the adult prostate epithelium. To test this hypothesis, we orthotopically transplanted dissociated mouse prostate organoid cells into immunocompetent syngeneic mice (Fig. 4a). Donor cells were injected into the extensive branchial structures of the distal anterior prostate, to avoid damage to the delicate proximal ducts, and maximize the probability of retention. Implantation of donor cells into an immunocompetent host tissue characterized by a slow turnover can be considered challenging. Still, we found that, in comparison to control organoid cells, organoid cells adapted to grow without A83-01 implanted with high frequency (Fig. 4b), and gave rise to dysplastic foci, characterized by elevated mitotic index, cuboidal histology and nuclear atypia (Fig. 4c,d).

We conclude that intra-epithelial non-canonical Activin A signalling safeguards quiescence in prostate progenitors.

\section{Discussion}

Signalling pathways ensure coordination of tissue development, homeostasis, regeneration,

211 and their disruption can lead to disease. The molecular bases of specific signals are difficult to 212 investigate, due to the challenges of disentangling cellular cross talks in vivo, and of establishing 213 representative models in vitro. More recently, organoid models in defined media conditions have 
opened new opportunities for the study of epithelia ${ }^{47}$. Benchmarking of these models with their corresponding in vivo counterpart is paramount for the correct experimental interpretations ${ }^{48}$. Here, we demonstrated that mouse prostate organoid cultures enable the continuous expansion of epithelial progenitors in vitro, including basal, LumP and PrU cells. Such cell types are predominantly found near the urethra in vivo, but also in the distal prostate compartment at low frequency. Our work revealed that progenitor proliferation is dynamically regulated by the antagonistic equilibrium between Egf and non-canonical Activin A signalling, respectively - with at least partial reduction of the latter required for cell cycle progression. The rationale perturbation of additional biochemical signals, and mechanical cues, may enhance progenitor differentiation towards distal luminal cells in culture.

It has long been known that the broad family of TGF- $\beta$ signals induces a cytostatic response in a large variety of epithelial cells, but the specific pathway acting in the prostate has remained poorly understood. Earlier studies pointed to the importance of the Tgf- $\beta$ family ligand Activin ${ }^{49,50}$. At the mechanistic level, DePinho and colleagues initially focused on the role of Smad4 as a proliferative barrier in a Pten-loss driven mouse model of prostate cancer ${ }^{51}$. Follow-up studies from the same group and others, carried out in humans and mice, have led to a more complex view ${ }^{52,53}$, with the involvement of both canonical and non-canonical pathways.

We propose a prominent role for non-canonical Activin A signalling in safeguarding quiescence in prostate epithelial progenitors (Fig. 4e). Our model may be relevant beyond tissue homeostasis and the response to inflammation. Genes encoding for core components of the noncanonical Activin A signalling pathway (e.g., $A C V R 2 A, M A P 3 K 7)$ are frequently lost in prostate cancer, based on large cohorts of patients in the U.S.A. ${ }^{54}$ and in China ${ }^{55}$. Moreover, MAP3K7 loss has been linked to genomic instability in human prostate cancer cell lines ${ }^{56}$ and found to promote an aggressive transcriptional programme in prostate tumours, based on a recent systematic pan-cancer analysis $^{57}$. In contrast, genetic alterations rarely affect TGFB1 receptors (e.g., TGFBR2) or SMAD factors (e.g., SMAD4), and enhanced canonical TGF- $\beta$ signalling has been reported in metastatic biopsies in therapy-resistant prostate cancer patients ${ }^{58}$. We speculate that loss of the non-canonical TGF- $\beta$ arm could impair the cytostatic response, while sparing the well-known transforming potential of the TGF- $\beta$ canonical pathway.

Tak1/p38-MAPK signalling stimulated two main sets of effector proteins in prostate progenitors. On the one hand, we observed the nuclear translocation of key negative cell cycle regulators (e.g., p16 and p21). On the other hand, using biochemical and transcriptional analyses, we demonstrated the activation of a broad transcriptional response, reminiscent of those induced by 
inflammatory cytokines and pathogens. These findings are in line with recent observations on the immune function of structural cells ${ }^{59}$ and suggest a cross talk between the epithelial and immune compartments, beyond the well-known mechanisms of anti-microbial defence. Prostate progenitors - and, perhaps, other types of epithelia cells - may have the ability to signal changes in their proliferative status and, immune cells may have the capability to adjust their function in response.

To test the relevance of intra-epithelial non-canonical Activin A signalling for the enforcement of quiescence in prostate progenitors, we performed experiments in vitro and in vivo. Our long-term organoid cultures - in the absence of Tgf- $\beta$ receptor blockade - revealed that cells capable to re-enter cell cycle had downregulated Activin A secretion. Moreover, those cells were also capable to implant and proliferate at increased frequency in vivo. While stromal sources of Tgf- $\beta$ ligands have been previously described in the prostate 25,26 , our study is the first to demonstrate a key role for intra-epithelial signalling.

Notably, dysregulation of non-canonical Activin A signalling was associated to DNA replication stress and genomic instability, a finding that may be relevant for tumour initiation. Indeed, distal LumP cells have been shown to serve as cell-of-origin for prostate cancer ${ }^{30}$. In this regard, our orthotopic transplantation approach may be particularly relevant for investigating the tumorigenic potential of distal progenitor cells.

Finally, P38 MAPK inhibitors - including Ralimetinib - are currently being tested in phase $1 / 2$ clinical trials ${ }^{60}$. Quiescent tumour progenitors - induced by the broad family of TGF- $\beta$ signals are emerging as key mediators of chemotherapy resistance in solid malignancy ${ }^{61}$. In advanced prostate cancers with a genetically intact Activin A non-canonical pathway, P38 MAPK inhibitors may force tumours progenitors out of quiescence, improving the efficacy of standard chemotherapy regimens ${ }^{62}$. While the complexity and pleiotropy of TGF- $\beta$ signalling has historically complicated drug development ${ }^{63}$, the elucidation of cell- and context-specific pathways may lead to novel therapeutic opportunities.

\section{Mice.}

274 Animal experiments were performed according to the European Communities Council Directive $275(2010 / 63 / \mathrm{EU})$ and approved by the Italian Ministry of Health and the University of Trento Animal 276 Welfare Committee (642/2017-PR). Wild-type C57BL/6J (JAX \# 000664) mice were purchased from 277 the Jackson Laboratory. Wild-type BALB/c (CRL \# 028) and CD-1 (CRL \# 022) mouse strains were obtained from the Charles River Laboratories. 


\section{Isolation of mouse prostate tissue.}

280 The anterior (AP), dorso-lateral (DLP) and ventral prostate (VP) lobes were dissected individually, 281 using a transverse cut at the intersection of each lobe with the urethra. Paired lobes were collected for 282 organoid cultures, histology and immunostaining studies.

\section{Dissociation of mouse prostate tissue to single cells.}

284 Prostate tissue was minced into small pieces, washed, resuspended into a digestion buffer -including 285 Collagenase II (1 mg/mL; Life Tech, 17101015) and Dispase II (10 mg/mL; Life Tech 17105041), 286 and transferred into a gentleMACS C tube (Miltenyi Biotec). Tissue fragments were processed by 287 alternating mechanical disruption - using the gentleMACS Dissociator (A.01 -C tube programme) 288 and enzymatic digestion -incubating the solution at $37^{\circ} \mathrm{C}$ on a tube rotator for 15 minutes. After three 289 cycles, the cell suspension was pelleted, resuspended in TrypLE (Life Tech, 12605010), and 290 incubated for 5 minutes at $37^{\circ} \mathrm{C}$. After two washes, the cell suspension was filtered through a $70 \mu \mathrm{m}$ 291 strainer and counted.

\section{3D prostate organoid cultures.}

293 Dissociated prostate cells were resuspended in $80 \%$ growth factor-reduced basement matrix (either 294 Matrigel ${ }^{\circledR}$, Corning, 356231; or BME-2 $\left.{ }^{\circ}, \mathrm{AMSBIO}, 3533\right)$ and seeded at the concentration of 295 approximately 50,000 cells $/ \mathrm{mL}$, by depositing at least six $40 \mu \mathrm{L}$ drops at the bottom of a non-tissue 296 culture treated plate. Basement matrix domes were left to solidify for 15 minutes and covered with 297 ENRAD medium - including Egf (50 ng/mL; PeproTech, 315-09), Noggin (100 ng/mL; PeproTech, 298 120-10C), R-Spondin1 (10\% conditioned medium), A83-01 (200 nM; Tocris, 2393) and 299 dihydrotestosterone (10 nM; Merck, 10300) - supplemented with Y-27632 (10 $\mu \mathrm{M}$; Calbiochem, 300 146986-50-7), as previously described ${ }^{64}$. Organoids were cultured in a standard tissue culture 301 incubator, with medium replacement every 2-3 days. After 6 days from the initial seeding, organoids 302 were imaged with a Leica MZ16F stereomicroscope and organoid forming efficiency was calculated. 303 For subsequent passages, the basement membrane was dissolved using a recovery solution 304 including Dispase II $(1 \mathrm{mg} / \mathrm{mL})$ - and organoids were dissociated to small clumps/single cells as 305 described above, using TrypLE. Following the first passage, organoids were seeded at the 306 concentration of approximately 25,000 cells $/ \mathrm{mL}$.

\section{Lentiviral transduction of organoids.}

308 Organoids were dissociated to single cells, and approximately 50,000 cells were transduced for each 309 condition. Spinoculation was performed in a low-adhesion 96 well-plate using 0.6 RTU of lentiviral 310 solution, supplemented with polybrene (4 $\mu \mathrm{g} / \mathrm{mL}$; Sigma Aldrich, H9268) and complete medium 311 (ENRAD) to reach a final reaction volume of $300 \mu \mathrm{L}$. The plate was sealed with parafilm and 
centrifuged for 1 hour at $600 \mathrm{~g}$. Afterwards, the cells were resuspended in $200 \mu \mathrm{L}$ of complete medium 313 (ENRAD), supplemented with Y-27632 $(10 \mu \mathrm{M})$, and incubated in suspension at $37^{\circ} \mathrm{C}$ for $4-6$ hour.

314 After centrifugation, the cell pellet was resuspended in $80 \%$ basement matrix and seeded as described 315 above. Antibiotic selection was initiated two days post-transduction. The following plasmids were 316 used: pLenti-AIB-EGFP (kindly donated by Massimo Pizzato), pSUPER-retro-puro-Smad4 317 (Addgene \#89829) and pSUPER-retro-puro-GFP shRNA (Addgene \#30519).

318 Treatments with growth factors and small molecule inhibitors.

319 Growth factors and small molecules used in this study are described in Supplementary Table 1.

320 Flow cytometry.

321 Organoids were dissociated to single cells as described above. For cell surface antigen expression 322 analysis, cells were incubated with anti-Cd24a-APC and Sca-1-PE-Cy7 antibodies (1:800 dilution) at $32344^{\circ} \mathrm{C}$ for 20-30 minutes, followed by one wash with FACS buffer (1\% FBS, 1mM EDTA). Cells were 324 resuspended in FACS buffer, supplemented with $1 \mu \mathrm{M}$ propidium iodide (Life Tech, P3566) for dead 325 cell exclusion, before proceeding to the analysis. For DNA content analysis, cells were resuspended 326 in $100 \mu \mathrm{l}$ ice-cold PBS and transferred to a $15 \mathrm{ml}$ tube. About $900 \mu \mathrm{l}$ of ice-cold EtOH $70 \%$ were 327 added dropwise while agitating the cell suspension on a vortex. Cells were then fixed for at least 2 328 hours at $-20^{\circ} \mathrm{C}$ before proceeding with 3 washing steps in PBS, alternated by centrifugation $(700 \mathrm{~g}, 5$ $329 \mathrm{~min})$ with no brake. Afterwards, cell pellet was resuspended in $100 \mu \mathrm{L}$ of DNAse-free RNAseA $(0.5$ $330 \mu \mathrm{g} / \mathrm{mL}$; Life Tech, 12091021) and incubated for $10 \mathrm{~min}$ at $37^{\circ} \mathrm{C}$. Cells were incubated with $100 \mu \mathrm{L}$ 331 of propidium iodide $(50 \mu \mathrm{g} / \mathrm{mL})$ for 30 minutes, at room temperature, before proceeding to the 332 analysis. For cell cycle analysis, organoids were treated with $10 \mu \mathrm{M}$ EdU for 3 hours. Afterwards, 333 organoids were harvested, dissociated into single cells, and filtered through a $30 \mu \mathrm{m}$ strainer. Cells 334 were pelleted and stained with the Click-iT ${ }^{\mathrm{TM}}$ Plus EdU Alexa Fluor ${ }^{\mathrm{TM}} 488$ Flow Cytometry Assay 335 Kit (Thermo Fisher Scientific, C10632), according to the manufacturer protocol. After the 336 incorporation of the fluorescent probe, cells were incubated with TO-PRO ${ }^{\text {TM}_{-}} 3$ Iodide (Life Tech, 337 T3695) to stain for DNA content, before proceeding to the analysis. Flow cytometry was performed 338 with a FACS Canto (BD) analyser, and data analysed with Flow Jo v.10.

339 Histology, immunostaining, and live imaging.

340 Organoids were cultured for 5-7 days, released from the basement membrane as described above, 341 seeded in a neutralized collagen type-I solution (Corning, 354249), and cultured for additional 24 342 hours, before proceeding to fixation in 4\% paraformaldehyde (Sigma Aldrich, P6148) for 5 hours, at 343 room temperature. Prostate tissue was harvested and immediately fixed using the same conditions. 344 Paraffin embedding and $5 \mu \mathrm{m}$ sectioning were carried out according to standard procedures. For 
immunofluorescence studies, antigen retrieval was performed using a citrate-based buffer ( $\mathrm{pH} 6.0)$ (Vector Lab, H3300). Slides were incubated in blocking solution (5\% FBS $+0.1 \%$ Triton-X in PBS), before proceeding to staining with primary antibodies, at $4^{\circ} \mathrm{C}$, overnight. After three washes, spectrally distinct fluorochrome-conjugated antibodies were incubated for 2 hours at room

temperature. After three additional washes, samples were counterstained with Hoechst 33342 (Abcam, ab145597), and the coverslip was applied, using FluorSave mounting medium (Merck, 345789). For immunohistochemistry studies, a similar protocol was followed, but using biotinconjugated secondary antibodies. The detection was performed using the Vectastain ${ }^{\circledR}$ Elite $A B C$ Peroxidase kit (Vector Labs, PK-6100) according to the manufacturer instructions. The final reaction was blocked by washing slides with water, and coverslips were applied using the DPX mounting medium (Sigma, 06522). For haematoxylin and eosin (H\&E) staining, deparaffinised sections were incubated with Gill haematoxylin (Merck, GH5232) for 2 minutes and washed with water. Samples were washed with ethanol, incubated with eosin Y (Merck, HT110132) for 3 minutes, washed again twice with ethanol, and treated with xylene, before mounting the coverslips in phenol based mounting medium. For immunostaining with anti-Egfr and anti-Acvrlb antibodies, the urogenital apparatus was isolated, snap-frozen in 2-methyl-buthanol cooled in liquid nitrogen, and cryo-sectioned at 20 $\mu \mathrm{m}$. Tissue slides were fixed in $4 \%$ paraformaldehyde for 20 minutes at room temperature, before proceeding as described above. For live imaging, organoids were stably transduced with pLenti-AIBEGFP. Images were acquired using either a Zeiss Axio Imager M2, or a Zeiss Axio Observer Z1 Apotome, or a Leica TCS SP8 Confocal. Image analysis was performed with the Zeiss ZEN software or ImageJ (v.2.0.0-rc-69/1.52i) ${ }^{65}$. Primary antibodies are listed in Supplementary Table 2.

\section{RNA extraction.}

367 Total RNA was extracted using the RNeasy Plus Micro kit (Qiagen, 74034) according to the manufacturer instructions, and analysed with an Agilent BioAnalyzer 2100 to confirm integrity (RIN $>8$ ), before proceeding with downstream applications.

\section{End-point semi-quantitative and quantitative real-time PCR.}

371 RNA was retrotranscribed into cDNA using the iScript ${ }^{\mathrm{TM}}$ cDNA synthesis kit (BioRad, 1708891). 372 End-point PCR was performed using Phusion Universal qPCR Kit (Life Tech, F566L), with PCR products visualized by standard gel electrophoresis. For quantitative real-time gene expression analysis, the qPCRBIO SyGreen Mix (PCRBiosystems, PB20.14-05) was used according to the manufacturer instructions. At least three independent biological replicates were run for each sample, using the CFX96 Real Time PCR thermocycler (Bio-Rad). The data were processed using Bio-Rad 
performed with GraphPad PRISM (v.6.01). Primer sequences are included in Supplementary Table 3.

\section{RNA sequencing and data analysis.}

381 cDNA libraries were prepared with TruSeq stranded mRNA library prep Kit (Illumina, RS-122-2101) 382 using $1 \mu \mathrm{g}$ of total RNA. RNA sequencing was performed on an Illumina HiSeq 2500 Sequencer 383 using standard Rapid Run conditions at the Next-Generation Sequence Facility of University of 384 Trento. The obtained reads were $100 \mathrm{bp}$ long, single ends, and 25 million on average for each sample. 385 FASTQ file from Illumina HiSeq2500 sequencing machine underwent adapter removal and quality386 base trimming using Trimmomatic-v0.35. Genomic alignments were performed onto the Mouse 387 genome (mm10 assembly version) using STAR-v2.6.0 aligner with a maximum mismatch of two and 388 default settings for all other parameters. Then, uniquely mapped reads were selected and processed 389 with HTSeq-count v0.5.4 tool to obtain gene-level raw counts based on GRCm38.92 Ensembl 390 (www.ensembl.org) annotation. Genes with CPM (Counts Per Million) $<1$ in all replicates were considered unexpressed and hence removed from the analysis. TMM (Trimmed Mean of M values) normalization and CPM conversion were next performed to obtain gene expression levels for downstream analyses. For each comparison, differential expression testing was performed using the edgeR-3.20.9 statistical package. According to the edgeR workflow, both common (all genes in all samples) and separate (gene-wise) dispersions were estimated and integrated into a Negative Binomial generalized linear model to moderate gene variability across samples. For each comparison, genes having a log Fold-change outside the range of $+/-1.5$ and a FDR q-value equal or smaller than 0.01 were considered as differentially expressed between the two groups.

\section{Gene Set Enrichment Analysis (GSEA).}

400 For the gene set enrichment analysis, the GSEA software (v4.0.3) was run in the 'pre-ranked' mode 401 using the Fold-change as a ranking metric and an FDR enrichment threshold of 0.25. Gene sets were 402 directly obtained from the Molecular Signature (MSig) database (http:// 403 software.broadinstitute.org/gsea/msigdb) focusing on all available sets reported in the following 404 MSigDB collections: C2 (curated gene sets): Biocarta, Kegg, Reactome; C5 (Gene ontology): 405 Biological Processes, Cellular Component, Molecular Function; C6 (oncogenic signatures) and C7 406 (immunologic signatures).

\section{Principal Component Analysis (PCA).}

408 PCA was performed using the DESeq2 R-package ${ }^{66}$ as follows: normalized counts (CPM) were firstly 409 converted into a DESeqDataset object through a DESeqDatasetFromMatrix function with default 410 parameters and transformed through the variantStabilizingTransformation function to stabilize 
411 variance-mean relation across samples. Then, transformed data was analyzed by PCA (plotPCA 412 function) generating a two-dimensional space where the two first components are represented.

\section{Subcellular Fractionation and Western blotting.}

414 Cell pellets from organoid cultures were obtained as previously described and lysed in fresh RIPA 415 buffer (50 mM Tris-HCl, pH 7.5, $150 \mathrm{mM} \mathrm{NaCl}, 1 \%$ Triton X-100, 1\% sodium deoxycholate, 1\% 416 NP-40) supplemented with protease (Halt ${ }^{\mathrm{TM}}$ protease inhibitor cocktail, Life Tech, 87786) and 417 phosphatase inhibitors (Phosphatase-Inhibitor Mix II solution, Serva, 3905501). Nuclear/cytoplasmic 418 fractionation was performed using NE-PER Nuclear and Cytoplasmic Extraction Kit (Life Tech, 419 78833) according to the manufacturer instructions. Protein concentrations were measured using the 420 BCA Protein Assay Kit (Pierce ${ }^{\mathrm{TM}}$ BCA Protein Assay kit, Thermo Fisher Scientific, 23225) and a 421 Tecan Infinite M200 Plate Reader.

422 Protein extracts were resolved via SDS-PAGE, transferred to polyvinylidene difluoride (PVDF) 423 membrane (Merck, GE10600023) using a wet electroblotting system (Bio-Rad). The membranes 424 were blocked with $5 \%$ non-fat dry milk or 5\% BSA in TBS-T (50 mM Tris-HCl, pH 7.5, $150 \mathrm{mM}$ $425 \mathrm{NaCl}, 0.1 \%$ Tween20) for 1 hour, at room temperature, and then incubated with gentle shaking with designated primary antibodies overnight, at $4^{\circ} \mathrm{C}$. Membranes were incubated with HRP-conjugated secondary antibody in blocking buffer for 1 hour at room temperature. Immunoreactive bands were detected using ECL LiteAblot plus kit A+B (Euroclone, GEHRPN2235) with an Alliance LD2 device and software (UVITEC). Primary antibodies are provided in Supplementary Table 4.

\section{Click-it chemistry-based mass spectrometry analysis.}

431 Organoids were seeded at the approximate concentration of 50,000 cells $/ \mathrm{ml}$, depositing seven $40 \mathrm{ul}$ 432 domes per individual well of a 6-well non-tissue culture plate. Three wells were used for each 433 condition. Following methionine depletion ( 2 hours), organoids were grown overnight at $37^{\circ} \mathrm{C}$ with 434 L-azidohomoalanine (AHA) medium. Conditioned supernatants were collected, supplemented with 435 protease inhibitors, and stored at $-80{ }^{\circ} \mathrm{C}$ until further processing. CLICK-IT enrichment of AHA436 labelled secreted proteins was performed with the Click-iT ${ }^{\mathrm{TM}}$ protein enrichment kit (Thermo Fisher 437 Scientific, C10416) as previously described ${ }^{67,68}$. Following trypsin digestion, peptides were purified 438 by reversed-phase (C18) stage-tip purification ${ }^{69}$. LC-MS/MS analysis was performed by an EASY439 LC 1000 coupled to a Q-Exactive mass spectrometer (Thermo Fisher Scientific). LC-MS/MS data 440 analysis was conducted using the MaxQuant/Perseus software suite.

441 Enzyme-linked immunosorbent assay (ELISA). 
Activin A quantification was performed using the corresponding Quantikine ELISA Kit (R\&D Systems, DAC00B) according to the manufacturer instructions.

\section{Karyotype analysis.}

445 Organoid cultures were treated with nocodazole (15 $\mu$ M; Sigma, SML1665) for 5 hours. Organoids 446 were recovered from basement membrane, and $900 \mu \mathrm{L} /$ sample of $50 \mathrm{mM} \mathrm{KCl}$ were added to the cell 447 pellet dropwise, followed by 10 minutes of incubation at $37{ }^{\circ} \mathrm{C}$. After centrifugation $(200 \mathrm{~g}, 5$ 448 minutes) $900 \mu \mathrm{L} /$ sample of Carnoy's fixative (methanol/acetic acid 3:1) was added dropwise. 449 Samples were resuspended and incubated for 10 minutes at $37{ }^{\circ} \mathrm{C}$, followed by three washes with 450 methanol/acetic acid 2:1. Approximately 25,000 cells/samples were resuspended in $50 \mu \mathrm{L}$ and 451 dropped from at least 1 meter of height, directly on a glass slide. After air-drying, the glass slide was 452 incubated with Hoechst 33342, at room temperature, for 10 minutes, and then washed with 453 methanol/acetic acid 2:1 for 5 minutes. After air-drying, coverslips were mounted with ProLong Gold 454 Antifade (Invitrogen, P36934). Images were acquired at the Zeiss Observer Z1 microscope and 455 analysed with ImageJ (v2.0.0-rc-69/1.52i) ${ }^{65}$.

456 Multicolor FISH (M-FISH), Chromosome painting and Telomeric FISH.

457 For M-FISH, fixed cells were dropped onto glass slides and hybridized with the 21XMouse 458 Multicolor FISH Probe Kit (MetaSystems, D-0425-060-DI), as previously described ${ }^{70}$. Briefly, the 459 slides were denatured in $0.07 \mathrm{~N} \mathrm{NaOH}$ and then rinsed in a graded ethanol series. The probe mix was 460 denatured using a MJ mini personal thermal cycler (Bio-Rad) with the following program: 5 minutes 461 at $75{ }^{\circ} \mathrm{C}, 30$ seconds at $10{ }^{\circ} \mathrm{C}$, and 30 minutes at $37^{\circ} \mathrm{C}$. The probe was added to the slides and the 462 coverslip was sealed using rubber cement. The samples were then hybridized in a humidified chamber 463 at $37{ }^{\circ} \mathrm{C}$ for $48 \mathrm{~h}$, washed in saline-sodium citrate (SSC) buffer for $5 \mathrm{~min}$ at $75{ }^{\circ} \mathrm{C}$, and finally 464 counterstained with DAPI (Abcam, 6843.2), in Vectashield mounting medium. Metaphases were 465 visualized and captured using a Zeiss Axio-Imager M1 microscope. The karyotyping and cytogenetic 466 analysis of each single chromosome was performed using the M-FISH module of the ISIS software 467 (MetaSystems). A total of 25 metaphases for each sample spreads were analysed in two independent 468 experiments.

469 For chromosome painting, fixed cells were dropped onto glass slides and hybridized with 470 enumeration XMP painting probes specific for chromosomes X (red label) and chromosome Y (green 471 label) (MetaSystems, D-1420-050-OR, D-1421-050-FI) following the manufacturer instructions. 472 Briefly, probes were applied to the slides, denatured at $75^{\circ} \mathrm{C}$ for 2 minutes, and then incubated at 37 $473{ }^{\circ} \mathrm{C}$ overnight. The slides were washed in SSC and counterstained with DAPI in antifade reagent 474 (MetaSystems, D-0902-500-DA). Metaphases were visualized and captured using a Zeiss Axio- 
475 Imager M1 microscope. A total of 100 metaphases were analysed for each sample in two independent experiments.

477 For telomeric FISH, staining was performed as previously described ${ }^{71}$. Briefly, slides and the Cy3 478 linked telomeric (TTAGGG)3 PNA probe, (DAKO Cytomatation, K5326) were co-denatured at 80 $479{ }^{\circ} \mathrm{C}$ for 3 minutes, and hybridized for 2 hours at room temperature, in a humidified chamber. After 480 hybridization, slides were washed and then dehydrated with an ethanol series and air dried. Finally, 481 slides were counterstained with DAPI and Vectashield. Images were captured at $63 \times$ magnification 482 using a Zeiss Axio-Imager M1 microscope, and the telomere signals were analysed using the ISIS 483 software (MetaSystems). Telomere doublets frequency was calculated as the ratio between the 484 number of doublets signals and the total number of chromosomes in each metaphase analysed ${ }^{72}$. At 485 least 20 metaphases in two independent experiments were analysed.

486 Orthotopic organoid transplantation.

487 Orthotopic transplantation of organoids into the prostate of syngeneic immune-competent C57BL/6J 488 mice was performed adapting a previously published method ${ }^{73}$. Organoids were dissociated as 489 described above, with 50,000 cells per injection resuspended in $10 \mu \mathrm{L}$ of $50 \%$ basement matrix, 490 supplemented with methylene blue (as tracer). Upon abdominal incision of the host, the left anterior 491 prostate lobe was exposed, and injected into the distal part. The contralateral lobe was injected with 492 saline as negative control. Mice were regularly monitored and sacrificed after 6 months for tissue 493 collection and histopathological analysis.

494 Statistical analysis and reproducibility.

495 No statistical methods were used to predetermine sample size. The in vitro experiments were not 496 randomized, and the investigators were not blinded to allocation during experiments and outcome 497 assessment. The in vivo transplantation experiments were randomized, and the investigators were 498 blinded to allocation during experiments and outcome assessment. The in vitro experiments were 499 carried out on organoid lines derived from at least two distinct animals and repeated at least three 500 independent times. The in vivo transplantation experiments were based on two distinct organoid lines 501 and were repeated at least two independent times. Data collection was performed using Microsoft 502 Office Excel 2016-2018 and statistical analysis was performed using GraphPad Prism 6 software. 503 The number of replicates, the format of the data, and the statistical tests are indicated in figure legends. 504 p-values $<0.05$ were considered significant.

\section{Data availability}


RNA sequencing datasets have been deposited on BioProject with the dataset identifier PRJNA659468. All other data supporting the findings of this study are available from the corresponding authors upon reasonable request.

\section{References}

1. De Marzo, A. M., Nelson, W. G., Meeker, A. K. \& Coffey, D. S. Stem cell features of benign and malignant prostate epithelial cells. The Journal of urology 160, 2381-92 (1998).

2. Toivanen, R. \& Shen, M. M. Prostate organogenesis: tissue induction, hormonal regulation and cell type specification. Development 144, 1382-1398 (2017).

3. Ørsted, D. D. \& Bojesen, S. E. The link between benign prostatic hyperplasia and prostate cancer. Nature Reviews Urology 10, 49-54 (2013).

4. Shinohara, D. B. et al. A mouse model of chronic prostatic inflammation using a human prostate cancer-derived isolate of Propionibacterium acnes. The Prostate 73, 1007-1015 (2013).

5. Kwon, O.-J., Zhang, L., Ittmann, M. M. \& Xin, L. Prostatic inflammation enhances basal-toluminal differentiation and accelerates initiation of prostate cancer with a basal cell origin. Proceedings of the National Academy of Sciences of the United States of America 111, E592600 (2014).

6. Simons, B. W. et al. A human prostatic bacterial isolate alters the prostatic microenvironment and accelerates prostate cancer progression. The Journal of Pathology 235, 478-489 (2015).

7. Kirby, R. S., Lowe, D., Bultitude, M. I. \& Shuttleworth, K. E. D. Intra-prostatic Urinary Reflux: an Aetiological Factor in Abacterial Prostatitis. British Journal of Urology 54, 729-731 (1982).

8. DuPre, N. C. et al. Corpora amylacea in prostatectomy tissue and associations with molecular, histological, and lifestyle factors. The Prostate 78, 1172-1180 (2018).

9. Nakai, Y., Nelson, W. G. \& Marzo, A. M. D. The Dietary Charred Meat Carcinogen 2-Amino1-Methyl-6-Phenylimidazo[4,5-b]Pyridine Acts as Both a Tumor Initiator and Promoter in the Rat Ventral Prostate. Cancer Res 67, 1378-1384 (2007).

10. Kwon, O.-J., Zhang, B., Zhang, L. \& Xin, L. High fat diet promotes prostatic basal-to-luminal differentiation and accelerates initiation of prostate epithelial hyperplasia originated from basal cells. Stem Cell Research 16, 682-691 (2016).

11. Zhang, B. et al. Non-Cell-Autonomous Regulation of Prostate Epithelial Homeostasis by Androgen Receptor. Molecular Cell 63, 976-989 (2016).

12. Wang, Z. et al. Androgenic to oestrogenic switch in the human adult prostate gland is regulated by epigenetic silencing of steroid 5 $\alpha$-reductase 2. The Journal of Pathology 243, 457-467 (2017).

13. Crowell, P. D. et al. Expansion of Luminal Progenitor Cells in the Aging Mouse and Human Prostate. Cell Reports 28, 1499-1510.e6 (2019).

14. Sfanos, K. S., Yegnasubramanian, S., Nelson, W. G. \& De Marzo, A. M. The inflammatory microenvironment and microbiome in prostate cancer development. Nature Reviews Urology 15, 11-24 (2018).

15. de Bono, J. S. et al. Prostate carcinogenesis: inflammatory storms. Nature Reviews Cancer 20, 455-469 (2020). 
16. Tucker, R. F., Shipley, G. D., Moses, H. L. \& Holley, R. W. Growth inhibitor from BSC-1 cells closely related to platelet type beta transforming growth factor. Science 226, 705-707 (1984).

17. Moses, H. L., Roberts, A. B. \& Derynck, R. The Discovery and Early Days of TGF- $\beta$ : A Historical Perspective. Cold Spring Harb Perspect Biol 8, a021865 (2016).

18. Mc Keehan, W. L. \& Adams, P. S. Heparin-binding growth factor/prostatropin attenuates inhibition of rat prostate tumor epithelial cell growth by transforming growth factor type beta. In Vitro Cell Dev Biol 24, 243-246 (1988).

19. Massagué, J. TGF $\beta$ signalling in context. Nature Reviews Molecular Cell Biology 13, 616-630 (2012).

20. Derynck, R. \& Budi, E. H. Specificity, versatility, and control of TGF- $\beta$ family signaling. Sci. Signal. 12, eaav5183 (2019).

21. Bailey, P. et al. Genomic analyses identify molecular subtypes of pancreatic cancer. Nature 531, 47-52 (2016).

22. Yaeger, R. et al. Clinical Sequencing Defines the Genomic Landscape of Metastatic Colorectal Cancer. Cancer Cell 33, 125-136.e3 (2018).

23. David, C. J. \& Massagué, J. Contextual determinants of TGF $\beta$ action in development, immunity and cancer. Nature Reviews Molecular Cell Biology 19, 419-435 (2018).

24. Gerstung, M. et al. The evolutionary history of 2,658 cancers. Nature 578, 122-128 (2020).

25. Salm, S. N. et al. TGF- $\beta$ maintains dormancy of prostatic stem cells in the proximal region of ducts. J Cell Biol 170, 81-90 (2005).

26. Wei, X. et al. Spatially Restricted Stromal Wnt Signaling Restrains Prostate Epithelial Progenitor Growth through Direct and Indirect Mechanisms. Cell Stem Cell 2, 24(5):753768.e6. (2019).

27. Henry, G. H. et al. A Cellular Anatomy of the Normal Adult Human Prostate and Prostatic Urethra. Cell Reports 25, 3530-3542.e5 (2018).

28. Karthaus, W. R. et al. Regenerative potential of prostate luminal cells revealed by single-cell analysis. Science 368, 497-505 (2020).

29. Joseph, D. B. et al. Urethral luminal epithelia are castration-insensitive cells of the proximal prostate. The Prostate 80, 872-884 (2020).

30. Guo, W. et al. Single-cell transcriptomics identifies a distinct luminal progenitor cell type in distal prostate invagination tips. Nature Genetics 52, 908-918 (2020).

31. Crowley, L. et al. A single-cell atlas of the mouse and human prostate reveals heterogeneity and conservation of epithelial progenitors. eLife 9, e59465 (2020).

32. Mevel, R. et al. RUNX1 marks a luminal castration resistant lineage established at the onset of prostate development. eLife 9, e60225 (2020).

33. Pignon, J.-C. et al. Cell Kinetic Studies Fail to Identify Sequentially Proliferating Progenitors as the Major Source of Epithelial Renewal in the Adult Murine Prostate. PLOS ONE 10, e0128489 (2015).

34. Kwon, O.-J. et al. The Sca-1+ and Sca-1- mouse prostatic luminal cell lineages are independently sustained. Stem Cells 38, 1479-1491 (2020).

35. Kwon, O.-J., Zhang, L. \& Xin, L. Stem Cell Antigen-1 Identifies a Distinct AndrogenIndependent Murine Prostatic Luminal Cell Lineage with Bipotent Potential. Stem Cells 34, 191-202 (2016). 
36. Karthaus, W. R. et al. Identification of multipotent luminal progenitor cells in human prostate organoid cultures. Cell 159, 163-175 (2014).

37. Chua, C. W. et al. Single luminal epithelial progenitors can generate prostate organoids in culture. Nature Cell Biology 16, 951-961 (2014).

38. Barros-Silva, J. D. et al. Single-Cell Analysis Identifies LY6D as a Marker Linking CastrationResistant Prostate Luminal Cells to Prostate Progenitors and Cancer. Cell Reports 25, 35043518.e6 (2018).

39. Sporn, M. B. \& Todaro, G. J. Autocrine Secretion and Malignant Transformation of Cells. $N$ Engl J Med 303, 878-80 (1980)

40. Eichelbaum, K., Winter, M., Diaz, M. B., Herzig, S. \& Krijgsveld, J. Selective enrichment of newly synthesized proteins for quantitative secretome analysis. Nature Biotechnology 30, 984 990 (2012).

41. Nakamura, T. et al. Activin-binding protein from rat ovary is follistatin. Science 247, 836-838 (1990).

42. Yamaguchi, K. et al. Identification of a Member of the MAPKKK Family as a Potential Mediator of TGF- $\beta$ Signal Transduction. Science 270, 2008-2011 (1995).

43. Shim, J.-H. et al. TAK1, but not TAB1 or TAB2, plays an essential role in multiple signaling pathways in vivo. Genes Dev. 19, 2668-2681 (2005).

44. Sato, S. et al. Essential function for the kinase TAK1 in innate and adaptive immune responses. Nature Immunology 6, 1087-1095 (2005).

45. Sorrentino, A. et al. The type I TGF- $\beta$ receptor engages TRAF6 to activate TAK1 in a receptor kinase-independent manner. Nature Cell Biology 10, 1199-1207 (2008).

46. Platanias, L. C. Mechanisms of type-I- and type-II-interferon-mediated signalling. Nature Reviews Immunology 5, 375-386 (2005).

47. Kretzschmar, K. \& Clevers, H. Organoids: Modeling Development and the Stem Cell Niche in a Dish. Developmental Cell 38, 590-600 (2016).

48. Huch, M., Knoblich, J. A., Lutolf, M. P. \& Martinez-Arias, A. The hope and the hype of organoid research. Development 144, 938-941 (2017).

49. Cancilla, B. et al. Regulation of Prostate Branching Morphogenesis by Activin A and Follistatin. Developmental Biology 237, 145-158 (2001).

50. Gold, E. \& Risbridger, G. Activins and activin antagonists in the prostate and prostate cancer. Molecular and Cellular Endocrinology 359, 107-112 (2012).

51. Ding, Z. et al. SMAD4-dependent barrier constrains prostate cancer growth and metastatic progression. Nature 470, 269-273 (2011).

52. Gerke, T. A. et al. Evaluating a 4-marker signature of aggressive prostate cancer using timedependent AUC. The Prostate 75, 1926-1933 (2015).

53. Lu, X. et al. Opposing roles of TGF $\beta$ and BMP signaling in prostate cancer development. Genes \& Development 31, 2337-2342 (2017).

54. Abeshouse, A. et al. The Molecular Taxonomy of Primary Prostate Cancer. Cell 163, 10111025 (2015).

55. Li, J. et al. A genomic and epigenomic atlas of prostate cancer in Asian populations. Nature 580, 93-99 (2020). 
56. Washino, S. et al. Loss of MAP3K7 Sensitizes Prostate Cancer Cells to CDK1/2 Inhibition and DNA Damage by Disrupting Homologous Recombination. Mol Cancer Res 17, 1985-1998 (2019).

57. Paull, E. O. et al. A modular master regulator landscape controls cancer transcriptional identity. Cell 184, 334-351.e20. (2021).

58. He, M. X. et al. Transcriptional mediators of treatment resistance in lethal prostate cancer. Nature Medicine 1-8 (2021).

59. Krausgruber, T. et al. Structural cells are key regulators of organ-specific immune responses. Nature 583, 296-302 (2020).

60. Vergote, I. et al. A randomized, double-blind, placebo-controlled phase $1 \mathrm{~b} / 2$ study of ralimetinib, a p38 MAPK inhibitor, plus gemcitabine and carboplatin versus gemcitabine and carboplatin for women with recurrent platinum-sensitive ovarian cancer. Gynecologic Oncology 156, 23-31 (2020).

61. Oshimori, N., Oristian, D. \& Fuchs, E. TGF- $\beta$ Promotes Heterogeneity and Drug Resistance in Squamous Cell Carcinoma. Cell 160, 963-976 (2015).

62. Quinn, D. I., Sandler, H. M., Horvath, L. G., Goldkorn, A. \& Eastham, J. A. The evolution of chemotherapy for the treatment of prostate cancer. Annals of Oncology 28, 2658-2669 (2017).

63. Akhurst, R. J. \& Hata, A. Targeting the TGF $\beta$ signalling pathway in disease. Nature Reviews Drug Discovery 11, 790-811 (2012).

64. Drost, J. et al. Organoid culture systems for prostate epithelial and cancer tissue. Nature Protocols 11, 347-358 (2016).

65. Rueden, C. T. et al. ImageJ2: ImageJ for the next generation of scientific image data. BMC Bioinformatics 18, 529 (2017).

66. Love, M. I., Huber, W. \& Anders, S. Moderated estimation of fold change and dispersion for RNA-seq data with DESeq2. Genome Biology 15, 550 (2014).

67. Eichelbaum, K., Winter, M., Diaz, M. B., Herzig, S. \& Krijgsveld, J. Selective enrichment of newly synthesized proteins for quantitative secretome analysis. Nature Biotechnology 30, 984 990 (2012).

68. Eihelbaum, K. \& Krijgsveld, J. Combining Pulsed SILAC labeling and Click-Chemistry for Quantitative Secretome Analysis. Methods in Molecular Biology 1174, 101-114 (2014).

69. Rappsilber, J., Mann, M. \& Ishihama, Y. Protocol for micro-purification, enrichment, prefractionation and storage of peptides for proteomics using StageTips. Nature Protocols 2, 18961906 (2007).

70. Nieri, D. et al. Cyogenetics effects in AG01522 human primary fibroblasts exposed to low doses of radiations with different quality. International Journal of Radiation Biology 89, 698707 (2013).

71. Samper, E., Goytisolo, F. A., Slijepcevic, P., van Buul, P. P. W. \& Blasco, M. A. Mammalian $\mathrm{Ku} 86$ protein prevents telomeric fusions independently of the length of TTAGGG repeats and the G-strand overhang. EMBO reports 1, 244-252 (2000).

72. Berardinelli, F. et al. G-quadruplex ligand RHPS4 radiosensitizes glioblastoma xenograft in vivo through a differential targeting of bulky differentiated- and stem-cancer cells. Journal of Experimental \& Clinical Cancer Research 38, 311 (2019).

73. O'Rourke, K. P. et al. Transplantation of engineered organoids enables rapid generation of metastatic mouse models of colorectal cancer. Nature Biotechnology 35, 577-582 (2017). 


\section{Acknowledgments}

676

We are grateful to Francesca Demichelis, Alberto Inga, Giannino del Sal, Marco Marcia, and Karuna Ganesh, for critical reading of the manuscript, and, Luciano Conti, Alessio Zippo, Luca Fava, Fulvio Chiacchiera, Luca Tiberi, Massimo Lopes, Alberto Briganti, Matteo Bellone and Marianna Kruithofde Julio for fruitful discussions. We thank Francesca Demichelis, Massimo Pizzato, Luca Fava, Yari Ciribilli, and Valeria Poli, for sharing reagents. We thank Veronica de Sanctis, Roberto Bertorelli and Paola Fassan of the Next Generation Sequencing Facility of the University of Trento for RNA sequencing. We also thank Sergio Robbiati and Marta Tarter of the CIBIO Model Organism Facility, Marina Cardano of the CIBIO Cell Technology Facility, Giorgina Scarduelli and Michela Roccuzzo of the CIBIO Advanced Imaging Facility, Isabella Pesce of the CIBIO Cell Analysis and Separation Facility, Valentina Adami and Michael Pancher of the CIBIO High-Throughput Screening Facility, and Isabelle Bonomo for assistance with experiments. We are grateful to Qingwen Jiang and Ahmed Mahmoud for help with illustrations, which were created using Inkscape and Biorender.com. This study was primarily supported by grants from the Giovanni Armenise-Harvard Foundation (Career Development Award to A.L.), the Lega Italiana Lotta ai Tumori (LILT-Bolzano to A.L.), the Italian Ministry for Research and the University (MIUR PRIN 2017 to A.L.), the Associazione Italiana per la Ricerca sul Cancro (AIRC MFAG 2017-ID 206221 to A.R.), the NIH (R01CA238005 to M.M.S.) - and by core funding from the University of Trento. Individual fellowships were awarded from the United States Department of Defense (W81XWH-18-1-0424 to F.C.), the European Union (H2020MSCA 749795 to A.Be.), the Fondazione Umberto Veronesi (FUV 2016 and 2017 to F.C., FUV 2016 to A.A., and FUV 2018 to A.Be.), and the University of Trento (Ph.D. fellowship to V.F, and D.D.F.).

\section{Author contributions}

F.C. made initial observations and designed the project in consultation with A.L.; F.C., V.F. and A.L. designed the experiments with the contribution of A.Ala. and D.D.F. regarding the Tak1/p38 MAPK signalling; F.C., with the contribution of M.Z., generated and characterized the normal prostate organoid lines described in this study; F.C., with the contribution of M.D.P., carried out the wet-lab based experiments defining the role of non-canonical Tgf- $\beta$ signalling in the control of epithelial progenitor proliferation and the ability of progenitors to spontaneously evade such regulatory mechanism; F.C., V.F., E.B. and D.D.F. characterized the consequences of non-canonical Tgf- $\beta$ signalling evasion in organoid cultures; V.F. generated the sh-Smad4 organoid line, with the contribution of D.D.F. and M.C., carried out the pharmacological studies on Tak1/p38 MAPK 
706 signalling; V.F., A.Ala., and D.D.F. performed biochemical studies on cell cycle regulators and 707 mediators of the type-I interferon-like response; V.F., D.D.F, S.G., G.M. and A.L. performed 708 orthotopic transplantations; M.L. and M.G. executed Click-it mass spectrometry experiments and 709 analysed the data; A.Be. contributed to flow cytometry; S.G. executed immunostaining experiments 710 and contributed to image acquisition; A.Bi. contributed to the characterization of p53 function in 711 organoids; F.B. and A.Ant. carried out and analysed chromosome painting and FISH studies; M.B., 712 M.F. and M.L provided histopathological annotations; F.G. and A.R. performed the computational 713 analyses; M.M.S. contributed to the interpretation of data; F.C. and V.F. assembled the figures; F.C. 714 wrote the first draft of the manuscript; F.C., V.F. and A.L. edited the manuscript with inputs from 715 F.B., A.Ant., A.R., M.L. and M.M.S.; A.L. acquired the main funding sources.

\section{Competing interests}

717 Authors declare no competing interests. 
bioRxiv preprint doi: https://doi.org/10.1101/2021.03.05.433921; this version posted March 13, 2021. The copyright holder for this preprint (which was not certified by peer review) is the author/funder, who has granted bioRxiv a license to display the preprint in perpetuity. It is made available under aCC-BY-NC-ND 4.0 International license.

Cambuli, Foletto, et al.
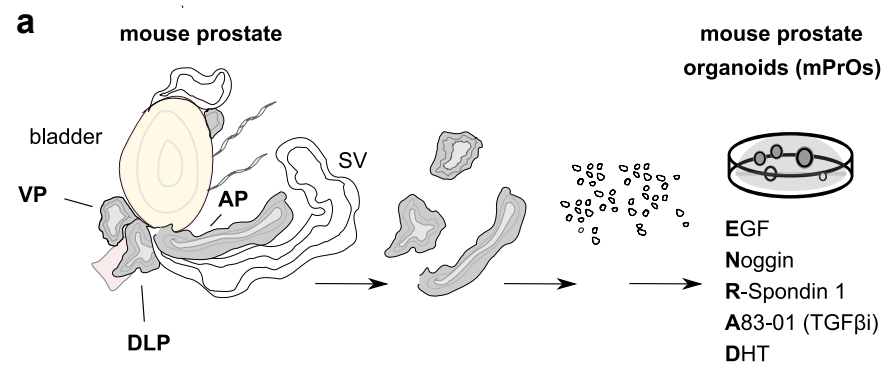

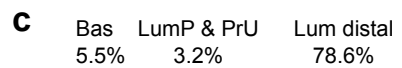
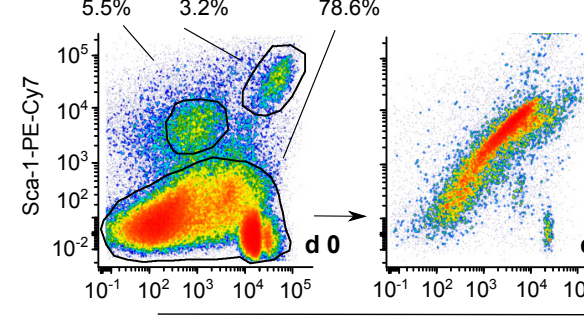

e
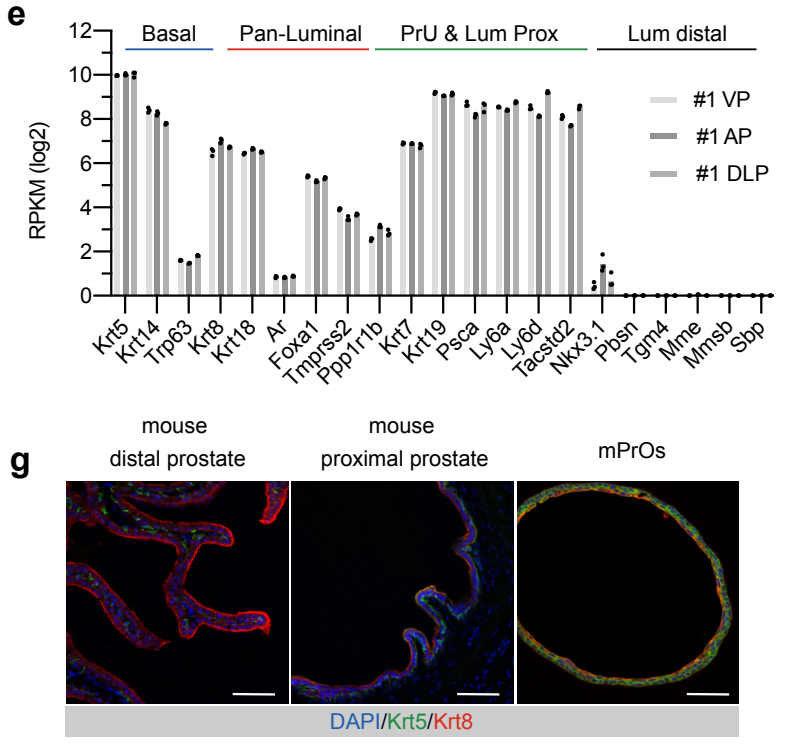

Figure 1
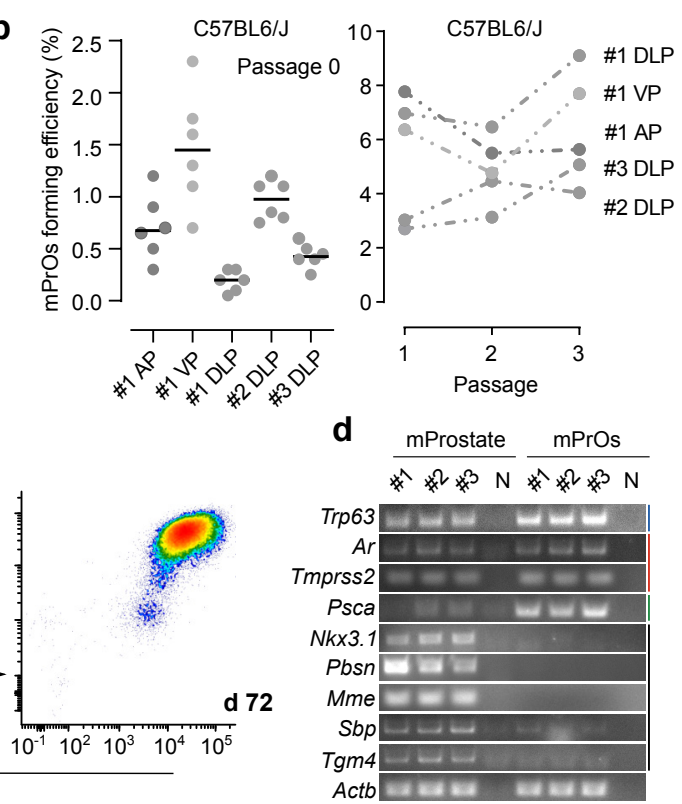

f
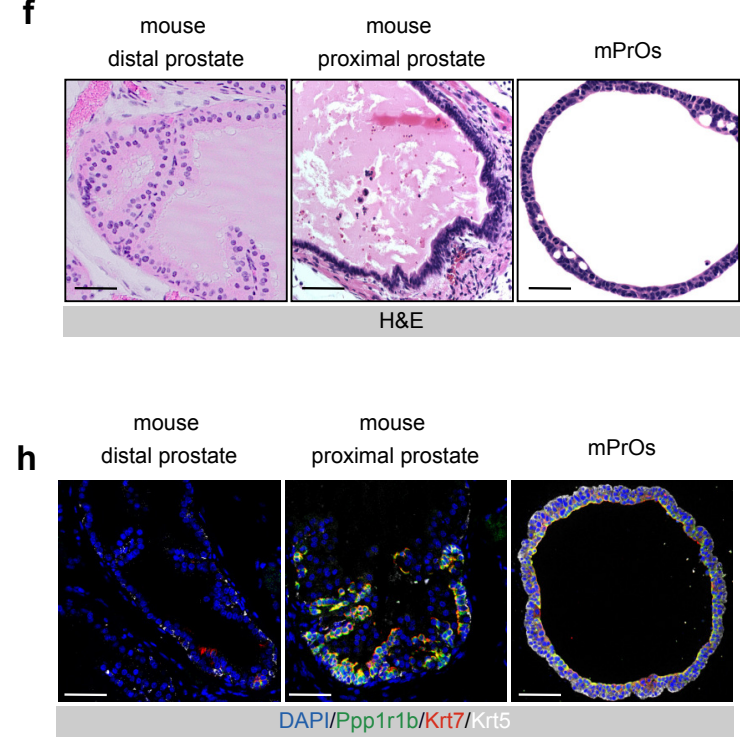

\section{Mouse prostate organoids are highly enriched in epithelial progenitor cells}

a. Schematic diagram describing organoid culture derivation (AP, Anterior Prostate; DLP, Dorso-Lateral Prostate; VP, Ventral Prostate; SV, Seminal Vesicle; DHT, dihydrotestosterone).

b. Mouse prostate organoid forming efficiency. Efficiency at derivation (left; data points are shown with crossing line representing mean value). Efficiency at passage 1-3 (right; $n \geq 3$ per organoid line/passage; data are presented as mean).

c. Representative longitudinal flow-cytometry analysis of dissociated organoid cells (Bas, Basal; LumP, Luminal Proximal; PrU, Periurethral; Lum Distal, Luminal Distal).

d. End-point RT-PCR analysis for selected marker genes.

e. Bulk-RNAseq analysis ( $n=3$; individual data points are shown with bar graphs representing mean value).

f. Representative Haematoxylin-Eosin (H\&E) staining of mouse prostate tissue and organoid sections (scale bars $=50 \mu \mathrm{m}$ ).

g. Representative Immunofluorescence (IF) analysis for selected markers in mouse prostate tissue and organoid sections (scale bars $=50 \mu \mathrm{m})$.

h. IF staining for selected markers in mouse prostate tissue and organoid sections (scale bars $=50 \mu \mathrm{m}$ ). 
Cambuli, Foletto, et al.

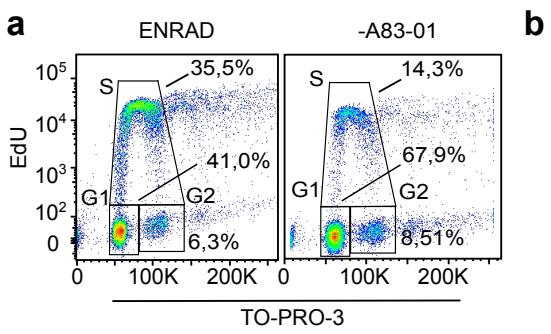

b

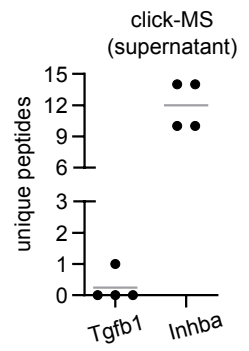

d

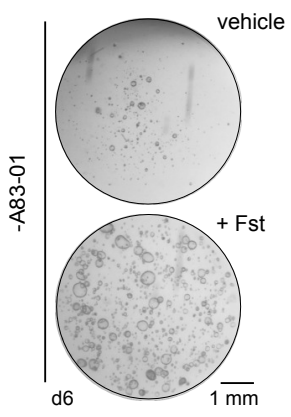

e

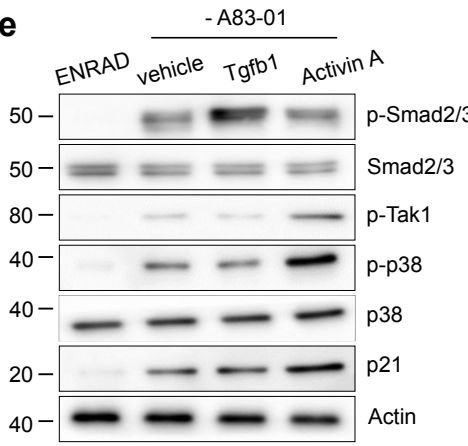

Figure 2
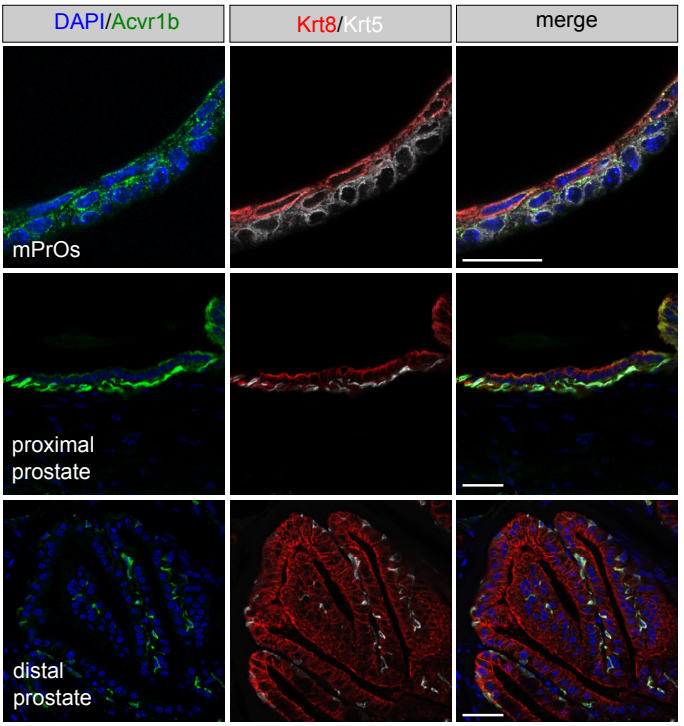

f

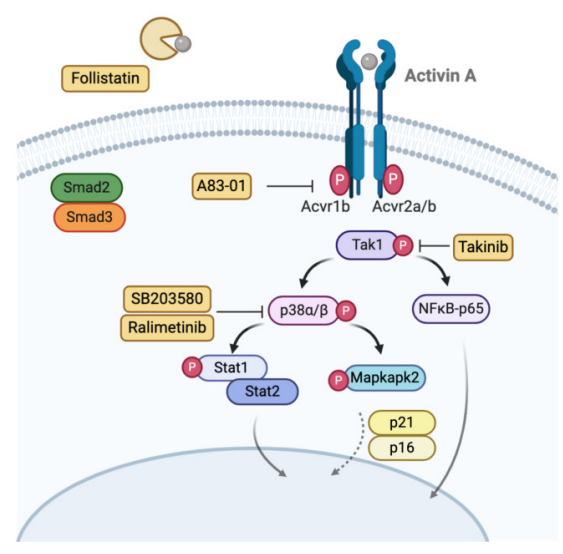

i

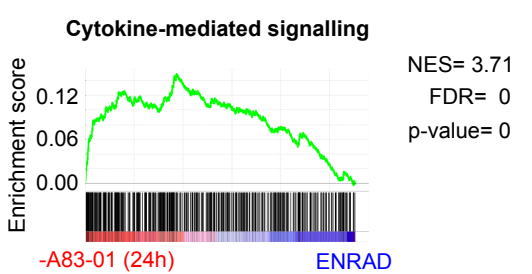

g

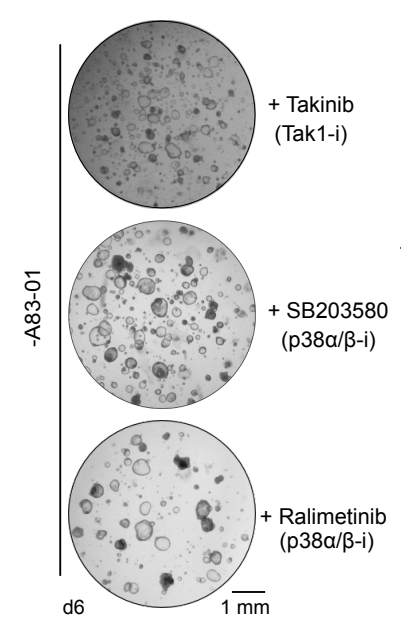

Tumor Necrosis Factor signalling

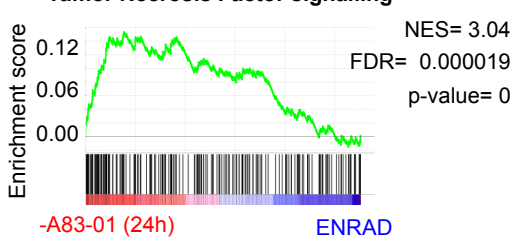

$\mathrm{ES}=3.04$

0.000019

ENRAD h
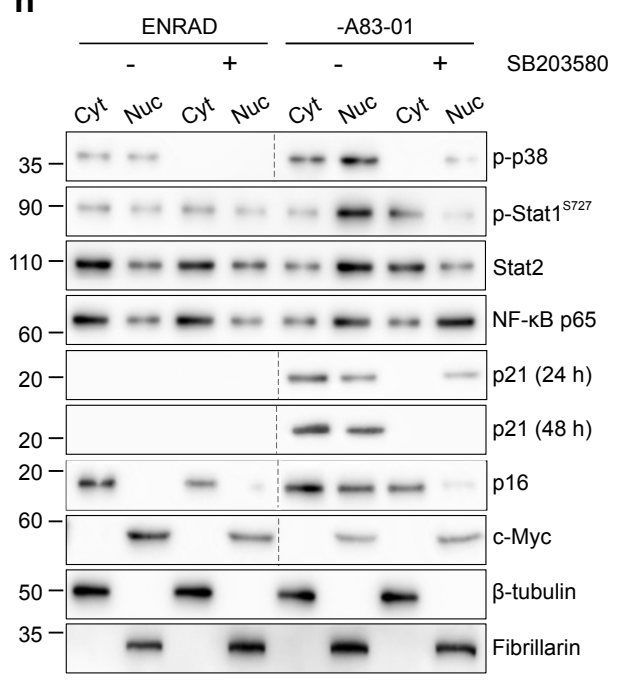

\section{Intra-epithelial non-canonical Activin A signalling mediates the Tgf- $\beta$ induced cytostatic response in mouse prostate organoids}

a. Cell cycle analysis by flow cytometry (EdU vs. TO-PRO-3) in complete medium (ENRAD) or in the absence of A83-01 (24 hours).

b. Detection of proteins secreted by mouse prostate organoids in culture based on click-chemistry enrichment and mass spectrometry analysis ( $n=4$; data points are shown with crossing line representing mean value).

c. IF analysis for selected markers in mouse prostate tissue and organoid sections (scale bar $=50 \mu \mathrm{m}$ ).

d. Representative stereoscopic images of mouse prostate organoids cultured in the absence of A83-01, with or without Follistatin (Fst, $500 \mathrm{ng} / \mathrm{mL}, 6$ days).

e. Western blot (WB) analysis in mouse prostate organoids for selected canonical and non-canonical Tgf- $\beta$ signalling mediators, and the cell cycle inhibitor p21 (Activin A, $50 \mathrm{ng} / \mathrm{mL}$; Tgfb1, $500 \mathrm{ng} / \mathrm{mL} ; 24$ hours).

f. Schematic view of the non-canonical Activin A pathway, including inhibitors used for the experiments described in this figure.

g. Representative stereoscopic images of mouse prostate organoids following treatment with Takinib (Tak1 inhibitor; $5 \mu \mathrm{M}, 6$ days), SB203580 ( $\mathrm{p} 38 \alpha / \beta$ inhibitor; $10 \mu \mathrm{M}, 6$ days) or the structurally unrelated Ralimetinib ( $\mathrm{p} 38 \alpha / \beta$ inhibitor; $1 \mu \mathrm{M}, 7$ days).

h. Nuclear fractionation and WB analysis in mouse prostate organoids for selected signalling mediators and cell cycle regulators, in the presence or absence of SB203580 (p38 $\alpha / \beta$ inhibitor; $10 \mu \mathrm{M}, 24$ hours).

i. Gene set enrichment analysis (GSEA) plots displaying significant enrichment for cytokine-mediated and Tumour Necrosis Factor (TNF) signalling in mouse prostate organoids cultured without A83-01 (24 hours) versus complete medium (ENRAD). 
Cambuli, Foletto, et al.

a

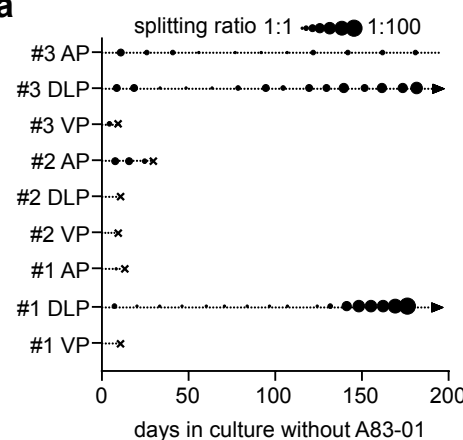

C

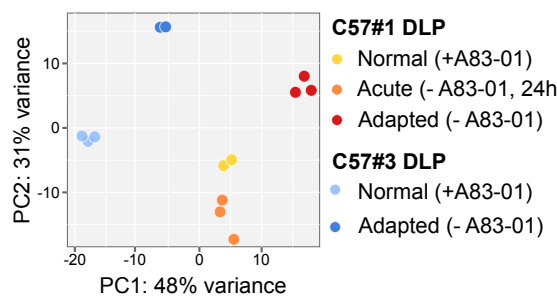

f

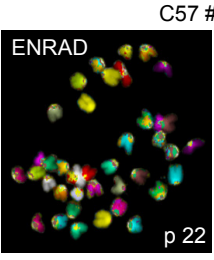

i

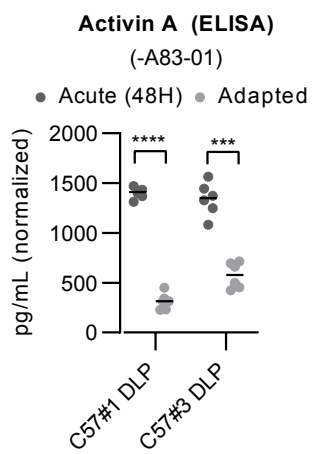

j

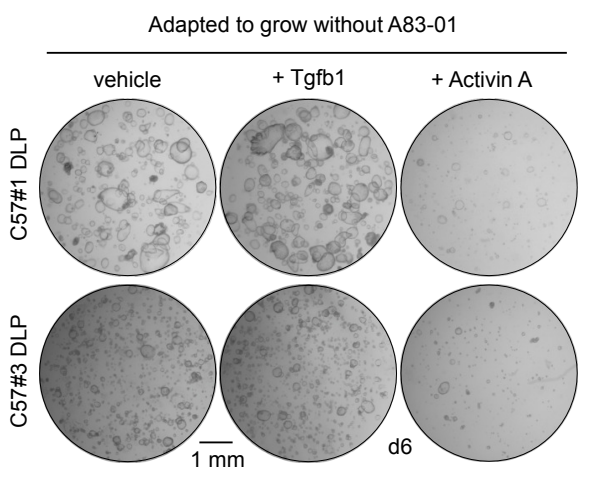

Figure 3

b

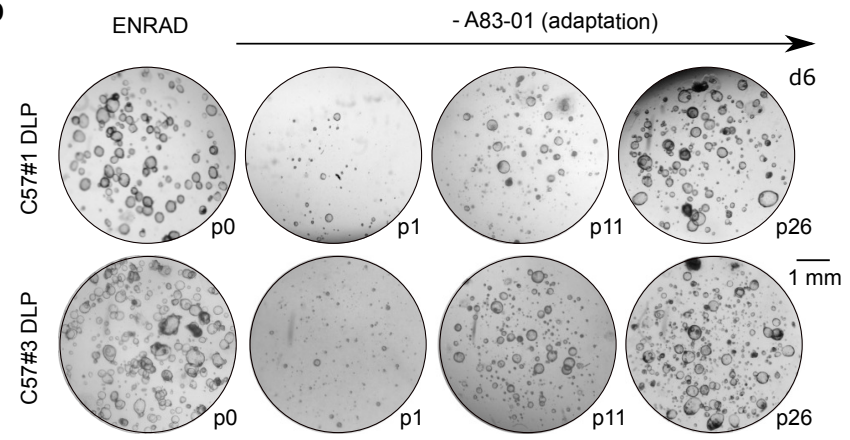

d ATR activation in response to DNA replication stress
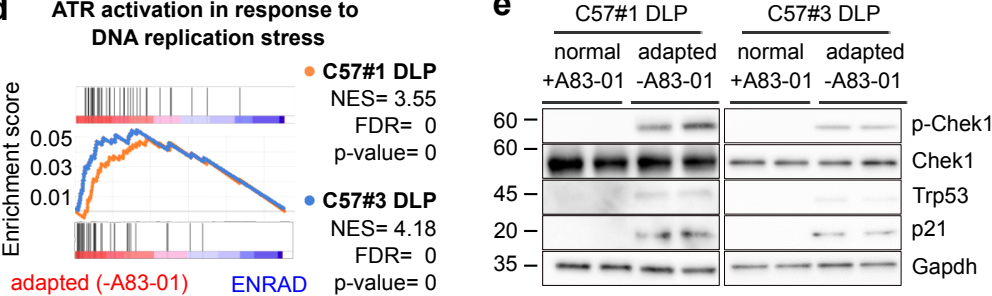

h

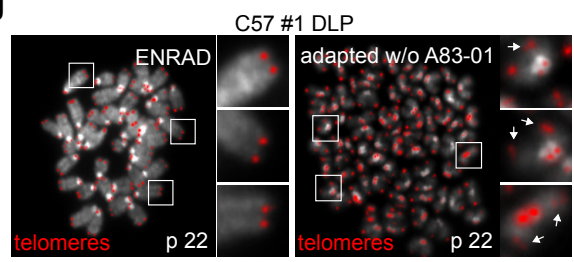

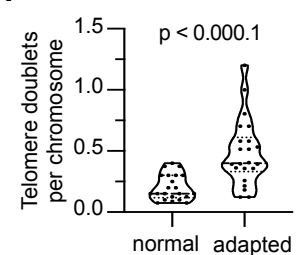

$\mathbf{k}$

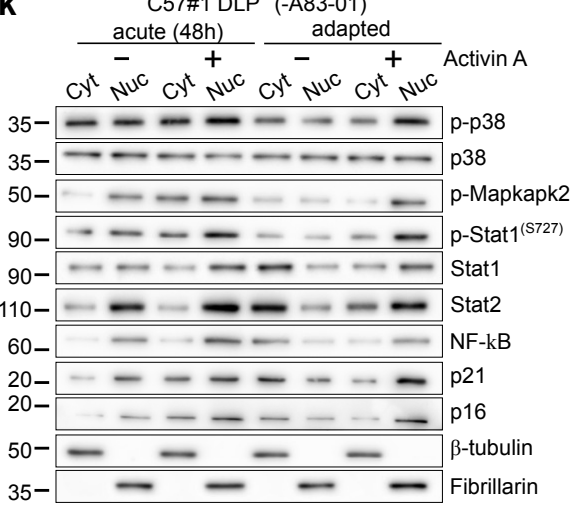

\section{Evasion from the Tgf- $\beta$ induced cytostatic response occurs via downregulation of intra-epithelial Activin A signalling and leads to DNA replication stress and genomic instability.}

a. Diagram depicting the expansion of mouse prostate organoid cultures in the absence of the Tgf- $\beta$ ligand inhibitor A83-01 (arrow $=$ continuous expansion; dot = passage; cross = culture loss).

b. Representative stereoscopic images of C57BL/6J DLP \#1 and \# 3 mouse prostate organoid lines during adaptation in the absence of A83-01.

c. Principal Component Analysis (PCA) based on bulk RNA-sequencing of C57\#1 DLP and C57\#3 DLP mouse prostate organoids grown in normal conditions (ENRAD), upon acute A83-01 depletion (-A83-01, 24 hours) (C57\#1 DLP only) or following adaptation (-A83-01, long-term).

d. Gene set enrichment analysis (GSEA) plot displaying significant enrichment for activation of ATR signalling in mouse prostate organoid lines (C57\#1 DLP, top; C57\#3 DLP, bottom) adapted to grow in the absence of A83-01 vs. normal control organoids cultured in complete medium (ENRAD).

e. WB analysis in C57\#1 and C57\# 3 DLP mouse prostate organoid lines adapted to grow in the absence of A83-01 vs. normal control organoids cultured in complete medium (ENRAD). Immunoblots are displayed for Chek1 (ATR signalling mediator), Trp53 and p21.

f. Representative spectral karyotype (SKY) images of metaphase spreads obtained from C57\#1 DLP mouse prostate organoids cultured in normal conditions (ENRAD) or upon adaptation without A83-01. Widespread genomic instability is observed following adaptation.

g-h. Representative telomere FISH images - and quantification - in C57\#1 DLP mouse prostate organoids cultured in normal conditions (ENRAD) or upon adaptation without A83-01. Widespread telomeric instability is observed following adaptation.

i. Enzyme-linked immunosorbent assay (ELISA) for Activin A expression in the supernatant of C57\#1 and C57\# 3 DLP mouse prostate organoid lines, upon acute A83-01 removal (48 hours) or long-term adaptation. Two-way ANOVA, Sidak's test, $p$-value ${ }^{* * *}(<0.001),{ }^{* * * *}(<0.0001)$.

j. Representative stereoscopic images of C57\#1 and C57\# 3 DLP mouse prostate organoid lines adapted to the absence of A83-01 and subsequently treated with either Tgfb1 ( $500 \mathrm{ng} / \mathrm{mL}, 6$ days) or Activin A (50 ng/mL, 6 days).

k. WB analysis in the C57\#1 DLP mouse prostate organoid line upon acute removal (24 hours) or long-term adaptation to A83-01 removal, in the presence or absence of Activin A ( $50 \mathrm{ng} / \mathrm{mL}, 24$ hours). 
bioRxiv preprint doi: https://doi.org/10.1101/2021.03.05.433921; this version posted March 13, 2021. The copyright holder for this preprint (which was not certified by peer review) is the author/funder, who has granted bioRxiv a license to display the preprint in perpetuity. It is made available under aCC-BY-NC-ND 4.0 International license.

Cambuli, Foletto, et al.

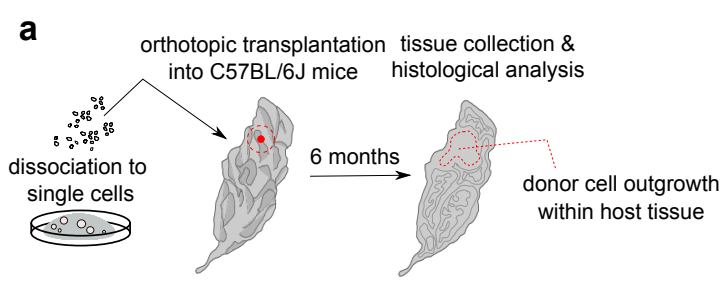

d
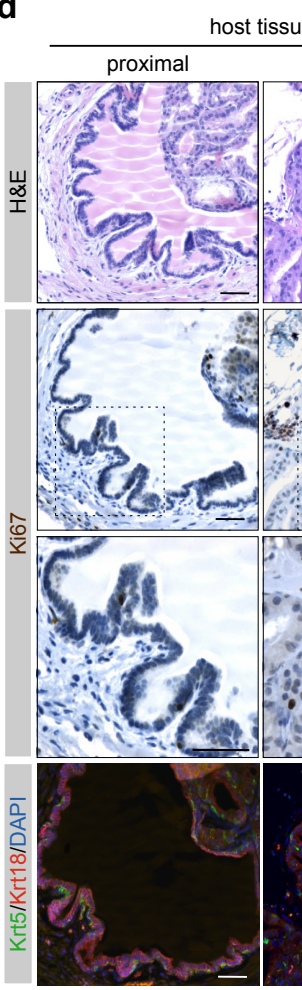

host tissue distal normal
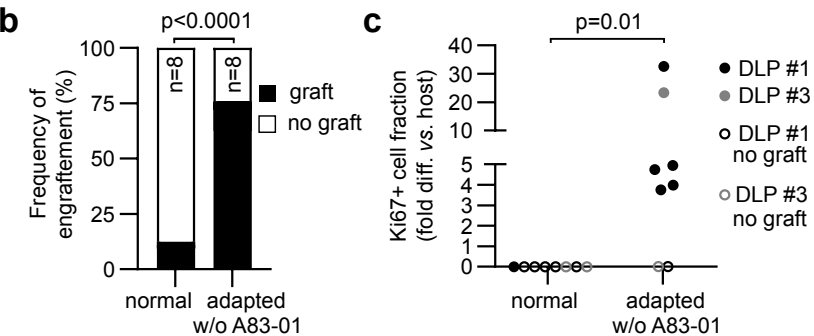

engrafted donor organoid cells adapted w/o A83-01
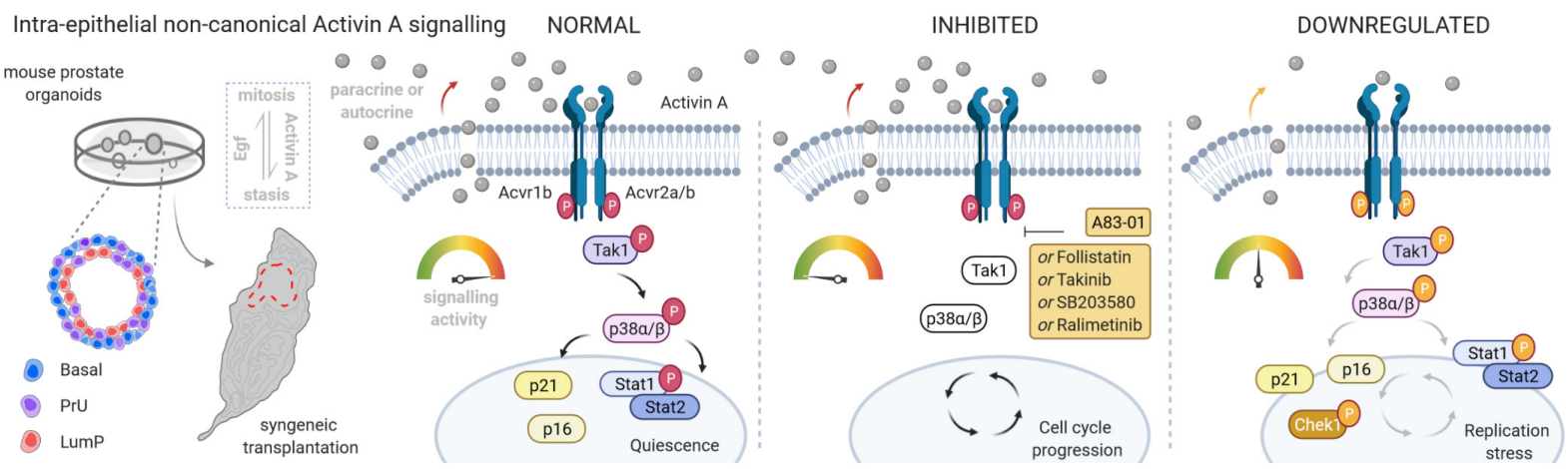

\section{Enhanced engraftment of mouse prostate organoids with reduced Activin A signalling into immunocompetent hosts.}

a. Schematic view of the orthotopic transplantation strategy into the anterior prostate lobe of immunocompetent syngeneic C57BL/6J mice.

b. Frequency of engraftment (\%) of mouse prostate organoid lines (C57\#1 DLP and C57\#3 DLP) expanded in complete medium (normal) or adapted to the absence of A83-01 (binomial test (two-tailed)).

c. Normalized mitotic (Ki67+) cell index in grafts of mouse prostate organoid lines (C57\#1 DLP and C57\# 3 DLP) expanded in complete medium conditions (normal) or adapted to the absence of A83-01 (non-parametric Mann-Whitney test).

d. Representative H\&E, IF, and immunohistochemistry (IHC) analyses of host prostate tissue and engrafted donor organoid cells (scale bar $=50 \mu \mathrm{m}$ ).

e. Intra-epithelial non-canonical Activin A signalling safeguards prostate progenitor quiescence. In normal circumstances, autocrine or paracrine Activin A triggers Tak1/p38 Mapk non-canonical signalling - antagonizing the pro-proliferative Egf pathway and enforcing cellular quiescence. Mechanistically, Tak1/p38 Mapk activity leads to p16 and p21 nuclear translocation - as well as to Stat1/2-dependent transcription of type-I interferon genes. Prostate organoid cultures require the suppression of this non-canonical signalling for continuous expansion, which can be achieved at different levels of the signalling cascade, using multiple inhibitors. Organoids with reduced levels on intra-epithelial Activin A signalling can be selected in vitro, and display enhanced engraftment efficiency in vivo, upon orthotopic transplantation into syngeneic hosts. Notably, the partial down-regulation of this pathway is associated with DNA replication stress and genomic instability. 
bioRxiv preprint doi: https://doi.org/10.1101/2021.03.05.433921; this version posted March 13, 2021. The copyright holder for this preprint (which was not certified by peer review) is the author/funder, who has granted bioRxiv a license to display the preprint in perpetuity. It is made available under aCC-BY-NC-ND 4.0 International license.

Cambuli, Foletto, et al.

a

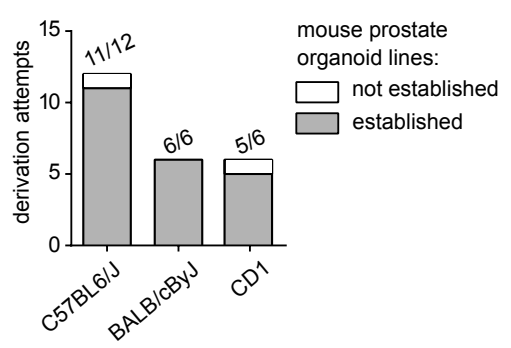

C

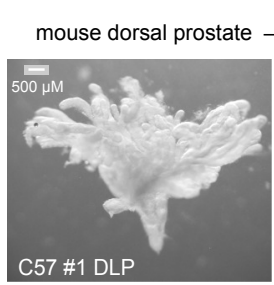

seeding of dissociated cells fully formed organoids

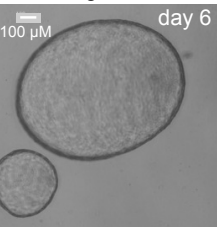

b

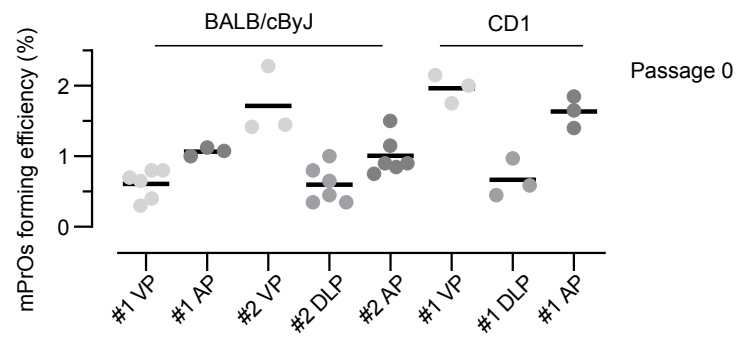

d

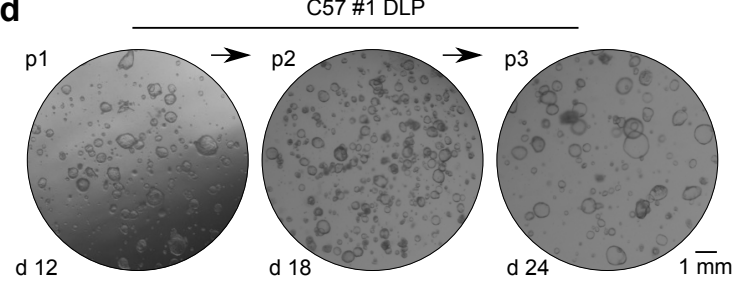

e

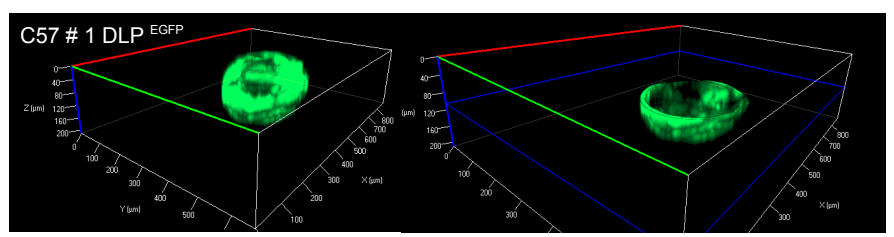

\section{Establishment of a mouse prostate organoid biobank}

a. Prostate organoid derivation rate from distinct mouse strains.

b. Mouse prostate organoid forming efficiency at derivation. Data points are shown with crossing line representing mean value.

c. Representative stereoscopic images at different stages of mouse prostate organoid derivation.

d. Representative stereoscopic images of mouse prostate organoid cultures during subsequent passages.

e. Representative 3D reconstruction of a fully formed mouse prostate organoid based on live epifluorescent microscopic analysis ( $1 \mu \mathrm{m}$ section $\times 300$ planes). On the right side, only the bottom half of the organoid is displayed. 
bioRxiv preprint doi: https://doi. org/10.1101/2021.03.05.433921; this version posted March 13, 2021. The copyright holder for this preprint (which was not certified by peer review) is the author/funder, who has granted bioRxiv a license to display the preprint in perpetuity. It is made available under aCC-BY-NC-ND 4.0 International license.

Cambuli, Foletto, et al.

a
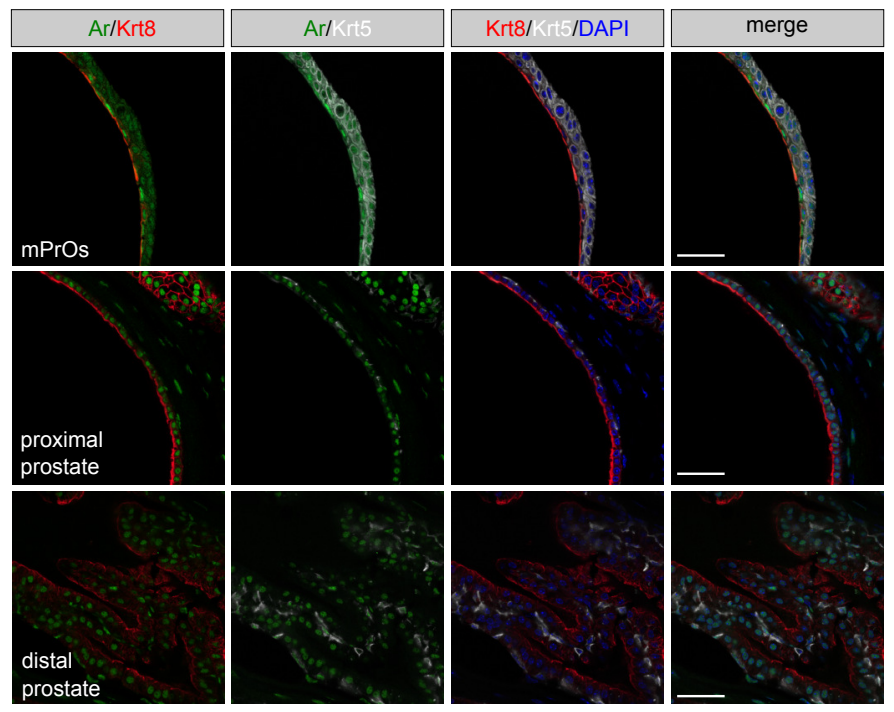

b

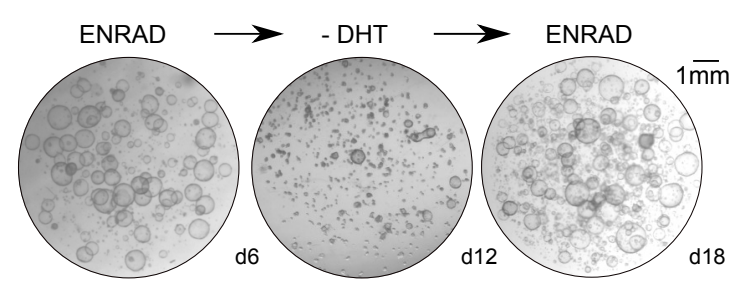

C

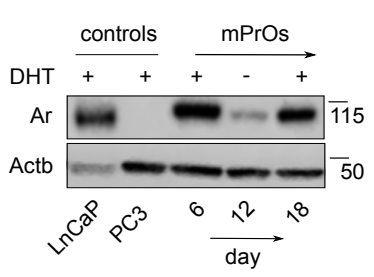

d

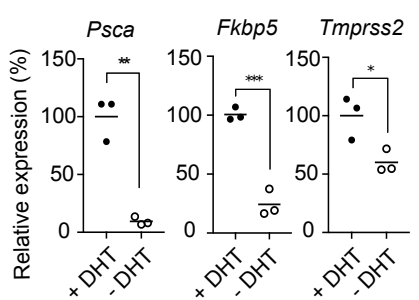

\section{Mouse prostate organoids are dependent on androgen signalling for lumen formation - but not for survival}

a. Immunofluorescence analysis for selected markers in mouse prostate tissue and organoid sections (scale bars $=50 \mu \mathrm{m}$ ).

b. Stereoscopic images of mouse prostate organoids experiencing transient dihydrotestosterone (DHT) removal (androgen cycling). Lumen formation necessitates androgen signaling.

c. Western blot analysis for Androgen receptor (Ar) expression in mouse prostate organoids experiencing androgen cycling, and control cell lines expressing ( $\mathrm{LnCaP}$ ) or not expressing (PC3) the receptor.

d. qRT-PCR mRNA expression analysis for selected androgen-responsive genes in the presence or absence of DHT. Data points are shown with crossing line representing mean value.

Student's t-test, two-tailed, p-value ${ }^{*}(<0.05),{ }^{* *}(<0.01),{ }^{* * *}(<0.001)$ 
bioRxiv preprint doi: $h$ ttps://doi. org/10.1101/2021.03.05.433921; this version posted March 13,2021 . The copyright holder for this preprint (which was not certified by peer review) is the author/funder, who has granted bioRxiv a license to display the preprint in perpetuity. It is made available under aCC-BY-NC-ND 4.0 International license.

Cambuli, Foletto, et al.

a

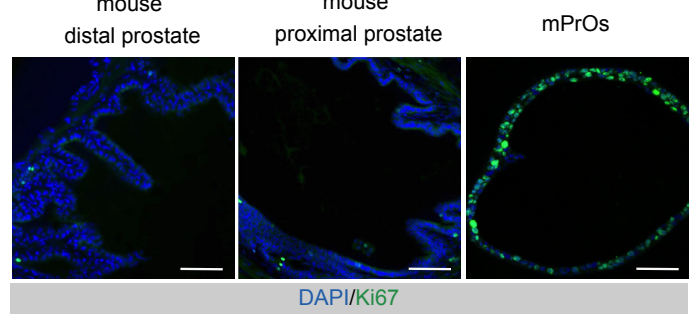

C

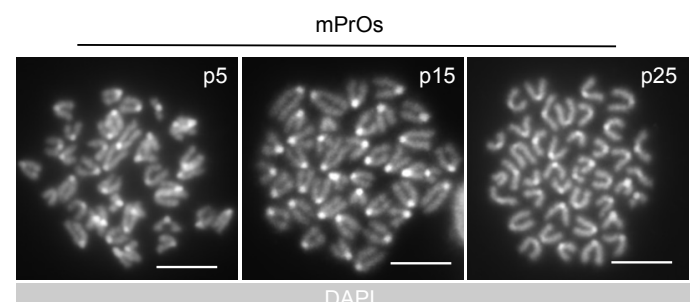

Supplementary Figure 3

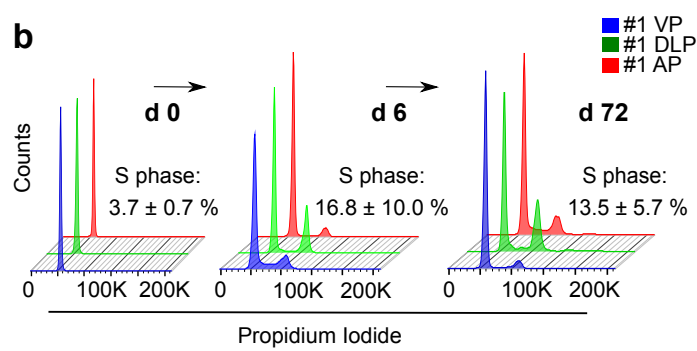

d

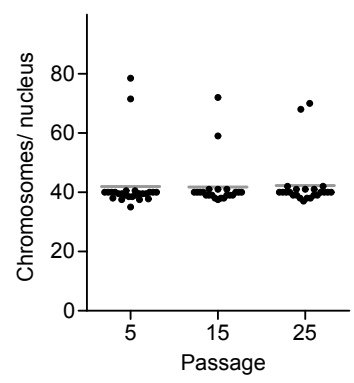

\section{Low levels of genomic instability in fast cycling mouse prostate organoids}

a. Immunofluorescence staining for Ki67 in mouse prostate tissue and organoid sections (scale bars $=50 \mu \mathrm{m}$ )

b. Flow cytometry analysis for DNA content

c. Representative karyotypes (scale bars $=10 \mu \mathrm{m}$ )

d. Quantification of karyotype analysis. Data points are shown with crossing line representing mean value. 
bioRxiv preprint doi: https://doi.org/10.1101/2021.03.05.433921; this version posted March 13, 2021. The copyright holder for this preprint (which was not certified by peer review) is the author/funder, who has granted bioRxiv a license to display the preprint in perpetuity. It is made available under aCC-BY-NC-ND 4.0 International license.

Cambuli, Foletto, et al.

Supplementary Figure 4

a
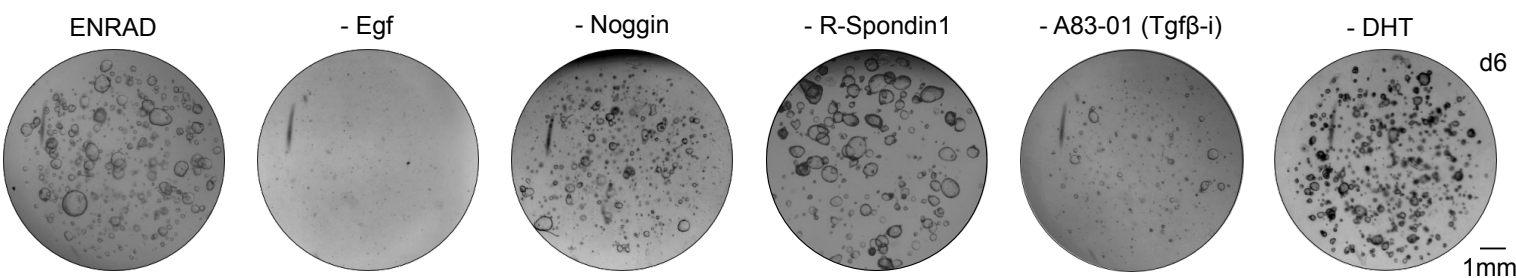

b

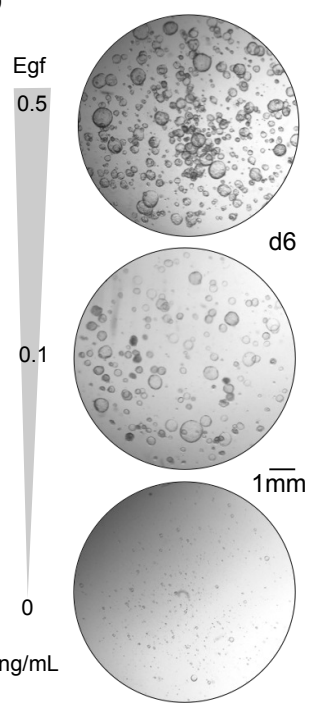

C

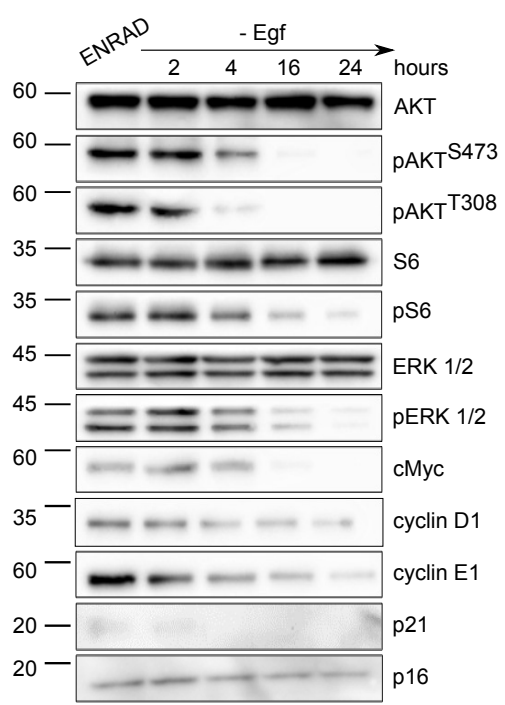

d

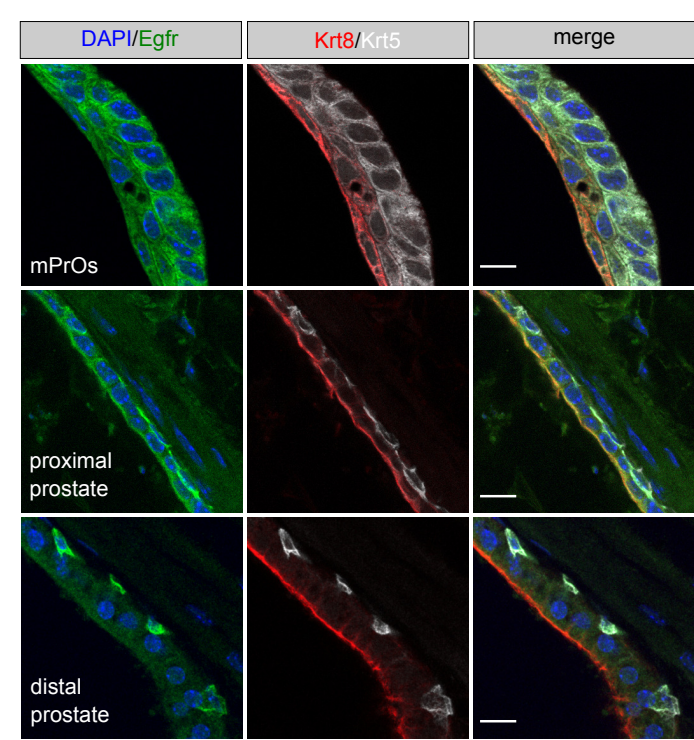

\section{Mouse prostate organoids rely on Egf signalling for continuous proliferation}

a. Representative stereoscopic images of mouse prostate organoid grown in complete medium (ENRAD) vs. medium depleted of individual growth factors/inhibitors. Mouse prostate organoids necessitate of both Egf and the Tgf- $\beta$ inhibiitor A83-01 for continuous expansion. Within the period of observation, removal of Noggin or R-Spondin 1 has no clear consequence in culture. Please note that organoids failed to form a lumen in the absence of dihydrotestosterone (DHT).

b. Stereoscopic images of mouse prostate organoids cultured in the presence of reduced levels $(0.5,0.1 \mathrm{ng} / \mathrm{mL})$ or in the absence of Egf.

c. Western blot analysis in mouse prostate organoids for selected signalling mediator and cell cycle regulator proteins.

d. Immunofluorescence analysis for selected markers in mouse prostate tissue and organoid sections (scale bars $=10 \mu \mathrm{m}$ ). 
bioRxiv preprint doi: https://doi.org/10.1101/2021.03.05.433921. this version posted March 13, 2021. The copyright holder for this preprint (which was not certified by peer review) is the author/funder, who has granted bioRxiv a license to display the preprint in perpetuity. It is made available under aCC-BY-NC-ND 4.0 International license.

Cambuli, Foletto, et al.

a

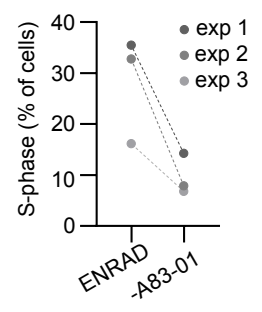

Supplementary Figure 5

b

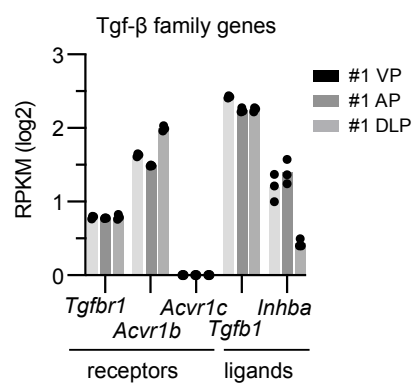

Inhibition of Tgf- $\beta$ receptors is required for cell cycle progression in mouse prostate organoids

a. Quantification of cells undergoing S-phase based on flow cytometry (EdU vs. TO-PRO-3)

in complete medium (ENRAD) or in the absence of A83-01 (24 hours). (Related to Fig 2a; n=3, individual data points are shown.)

b. mRNA expression levels for selected Tgf- $\beta$ family receptors and ligands. Bulk-RNAseq analysis

$(n=3$; individual data points are shown with bar graphs representing mean value). 
bioRxiv preprint doi: $h t t p s: / / d o i . o r g / 10.1101 / 2021.03 .05 .433921$; this version posted March 13,2021 . The copyright holder for this preprint (which was not certified by peer review) is the author/funder, who has granted bioRxiv a license to display the preprint in perpetuity. It is made available under aCC-BY-NC-ND 4.0 International license.

Cambuli, Foletto, et al.

Supplementary Figure 6

a

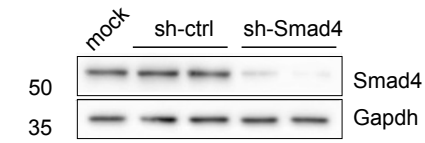

b

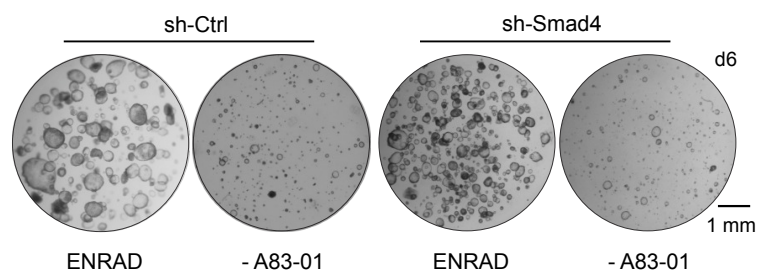

Canonical Smad signalling is dispensable for Tgf- $\beta$ induced quiescience in prostate organoids

a. Western blot analysis for Smad4 in mouse prostate organoids upon short-hairpin RNA mediated knockdown (sh-Smad4) and in control conditions (sh-Ctrl).

b. Representative stereoscopic images of control (sh-Ctrl) and Smad4 knocked down (sh-Smad4) mouse prostate organoids in normal growth condition (ENRAD) and following A83-01 withdrawal (-A83-01). 
Cambuli, Foletto, et al.

a

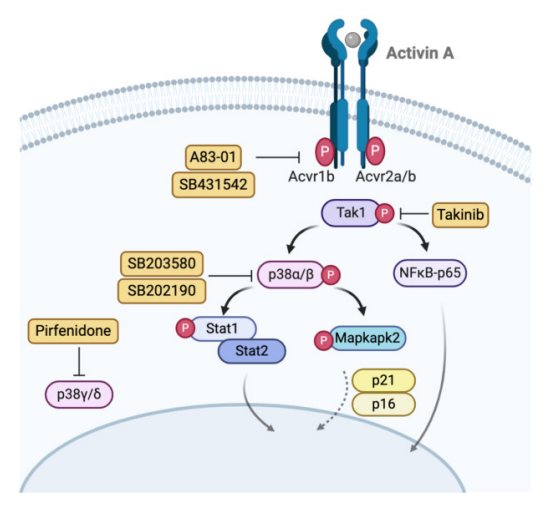

b

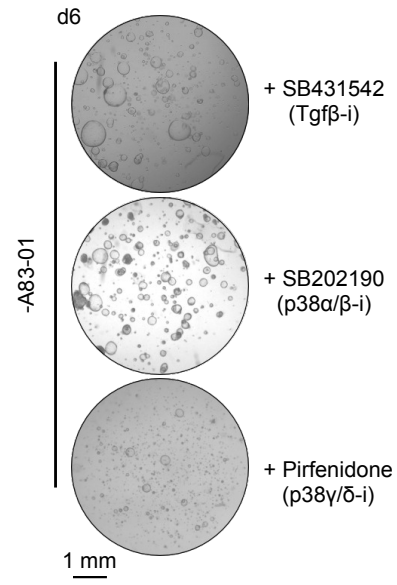

e

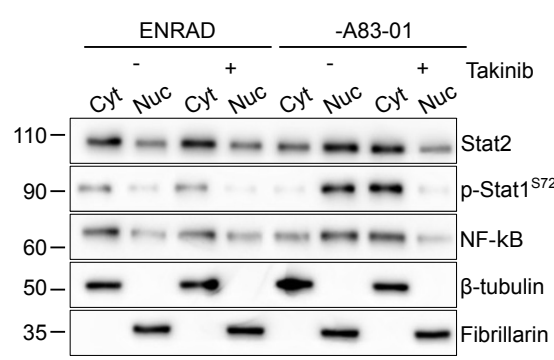

Supplementary Figure 7

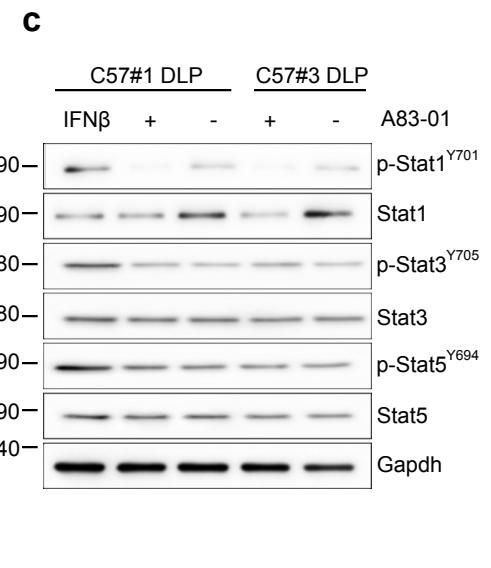

f

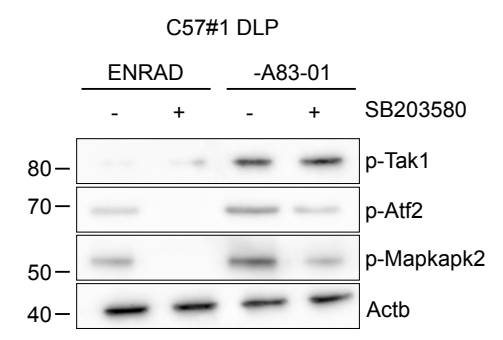

Tak1/p38 MAPK signalling activates immune- and stress-related pathways in prostate organoids

a. Schematic view of the non-canonical Activin A signalling pathwaty, including inhibitors used for the experiments described in this figure.

b. Representative stereoscopic images of mouse prostate organoids grown in the absence of A83-01 and in the presence of either SB431542 (Tgfß-receptor inhibitor; $10 \mu \mathrm{M}, 6$ days), or SB202190 (p38 $/ / \beta$ inhibitor; $10 \mu \mathrm{M}, 6$ days), or Pirfenidone (p38y/ठ inhibitor; $10 \mu \mathrm{M}, 6$ days).

c. Western blot analysis in mouse prostate organoids for canonical IFN signalling mediators upon acute A83-01 withdrawal (IFN $\beta ; 50 \mathrm{ng} / \mathrm{mL}, 6$ days).

d-f. Western blot analysis in mouse prostate organoids for key components of the non-canonical Tgf- $\beta$ pathway and IFN signalling upon acute A83-01 withdrawal and concomitant inhibition of either Tak1 (Takinib; $5 \mu \mathrm{M}, 24$ hours) (d, e) or $\mathrm{p} 38 \alpha / \beta$ (SB203580; $10 \mu \mathrm{M}, 24$ hours) (f). 
bioRxiv preprint doi: $h t t p s: / / d o i . o r g / 10.1101 / 2021.03 .05 .433921 \cdot$ this version posted March 13,2021 . The copyright holder for this preprint (which was not certified by peer review) is the author/funder, who has granted bioRxiv a license to display the preprint in perpetuity. It is made available under aCC-BY-NC-ND 4.0 International license.

Cambuli, Foletto, et al.

a

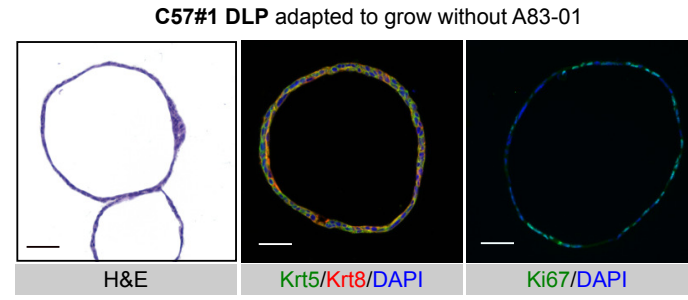

b

Supplementary Figure 8

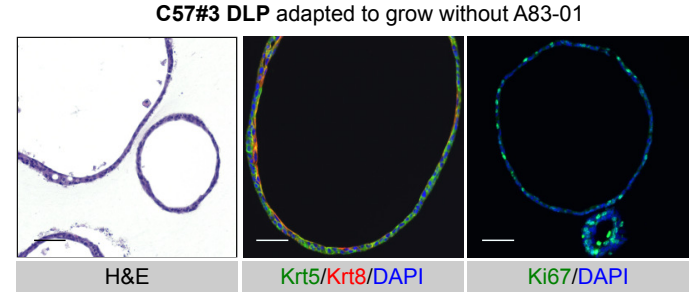

Mouse prostate organoids adapted to the absence of the Tgf-b receptor inhibitor A83-01 retain quasi-normal cellular identities and cytoarchitecture

a-b. Representative H\&E and immunofluorescence analyses of C57\#1 and C57\#3 DLP mouse prostate organoids upon adaptation to A83-01 withdrawal (scale bar $=50 \mu \mathrm{m}$ ). Please note the adapted organoids retain a pattern of cytokeratins expression consistent with normal cellular identities, including basal, luminal P (lumP) and peri-uretheral (PrU) 
bioRxiv preprint doi: https://doi.org/10.1101/2021.03.05.433921; this version posted March 13, 2021. The copyright holder for this preprint (which was not certified by peer review) is the author/funder, who has granted bioRxiv a license to display the preprint in perpetuity. It is made available under aCC-BY-NC-ND 4.0 International license.

Cambuli, Foletto, et al.

a

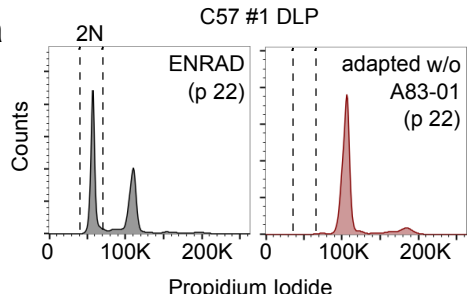

Supplementary Figure 9

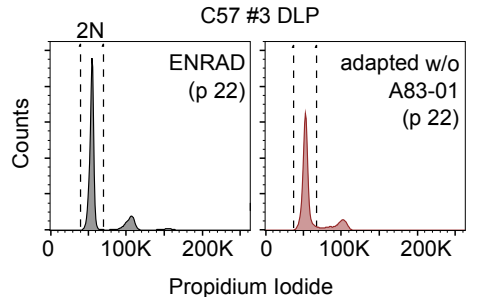

b
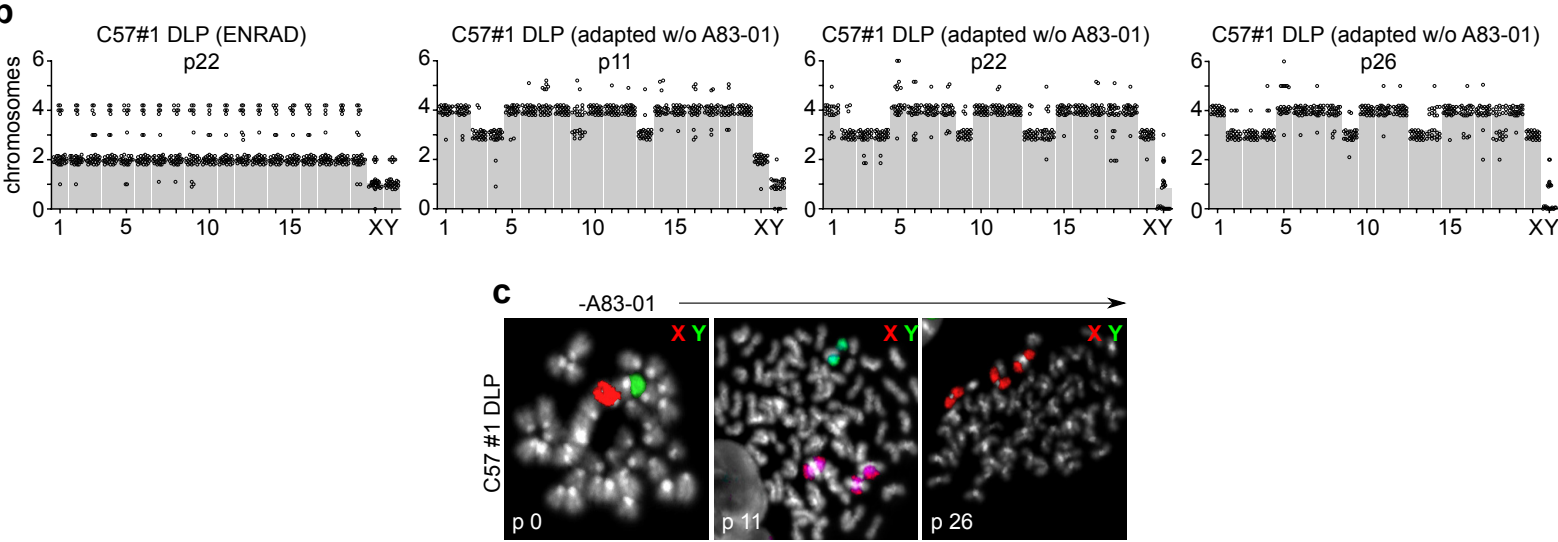
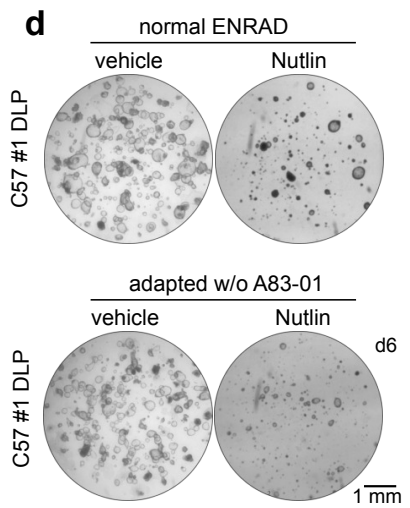
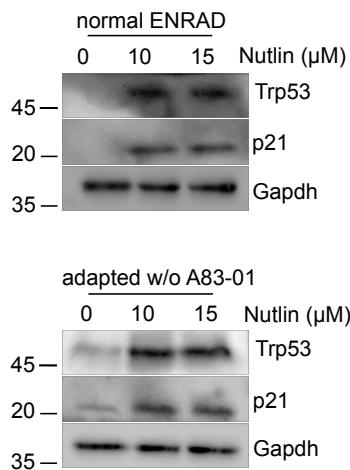

f

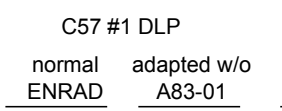
ENRAD A83-01

Doxorubicin $(\mu \mathrm{M})$

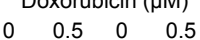

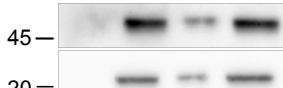

$20-$

$245-$

$245-7=$

$35----$
C57 \#1 DLP

normal adapted w/o ENRAD A83-01

$X$-rays (10 Gy) $0 \mathrm{~h} 8 \mathrm{~h} 0 \mathrm{~h} 8 \mathrm{~h}$

- - Trp53

$-\ldots$ p21

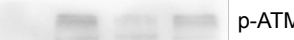

$\because-1=$ ATM

- - - Gapdh

\section{C57\#1 DLP mouse prostate organoids adapted to the absence of A83-01 in culture display widespread genomic instability while retaining an intact $\mathrm{p} 53$ pathway}

a. DNA content analysis in C57\#1 DLP and C57\#3 DLP mouse prostate organoid lines.

b. Quantification of spectral karyotype (SKY) longitudinal analysis in C57\#1 DLP mouse prostate organoids undergoing adaptation to the absence of A83-01 in culture (related to Fig 3f). During adaptation, an early genome duplication event is observed followed by selective chromosome loss and gains, and leading to subtetraploidy.

c. Representative dual-colour FISH images for $\mathrm{X}$ and $\mathrm{Y}$ chromosomes in C57\#1 DLP mouse prostate organoids during adaptation to the absence of A83-01 in culture. This analysis confirmed a progressive sex chromosome imbalance during adaptation.

d. Representative stereoscopic images of C57 \#1 DLP mouse prostate organoids treated with Nutlin, or vehicle as control.

e. Western blot analysis of C57 \#1 DLP mouse prostate organoids described in $\mathbf{d}$.

f. Western blot analysis in C57 \#1 DLP mouse prostate organoids treated with doxorubicin or X-rays. C57\#1 DLP mouse prostate organoids retain a functional p53 pathway. 
bioRxiv preprint doi: $h$ ttps://doi.org/10.1101/2021.03.05.433921; this version posted March 13, 2021. The copyright holder for this preprint (which was not certified by peer review) is the author/funder, who has granted bioRxiv a license to display the preprint in perpetuity. It is made available under aCC-BY-NC-ND 4.0 International license.

Cambuli, Foletto, et al.

a

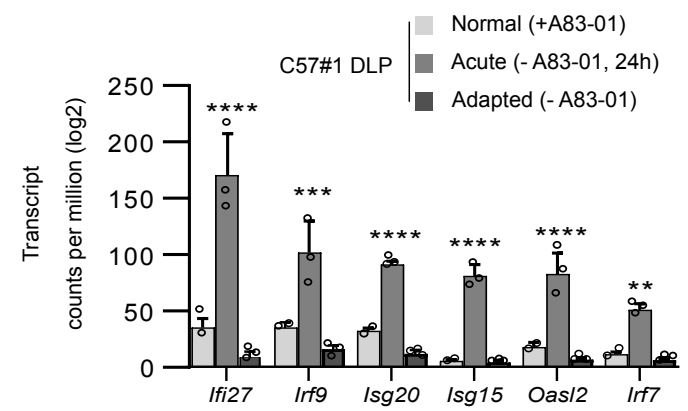

Supplementary Figure 10

b

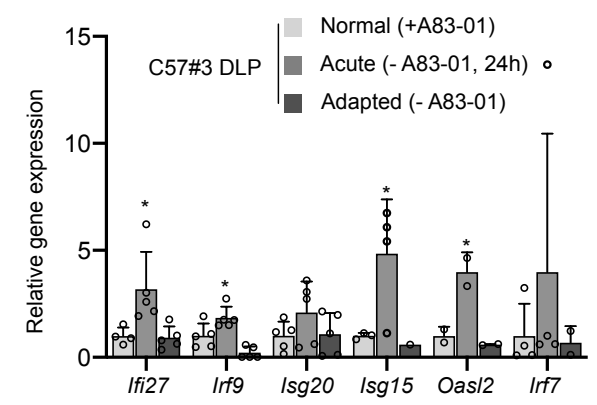

Non- canonical Activin A signalling regulates type-I interferon genes in mouse prostate organoids

a-b. mRNA expression levels for selected type-I IFN response genes in C57\#1 and C57\#3 DLP mouse prostate organoids cultured in complete medium (normal), upon acute A83-01 withdrawal (acute), and adapted to grow without A83-01 (adapted). Transcription of type-l interferon genes is enhanced upon A83-01 withdrawal and reduced upon adaptation ( $n=3$, bulk-RNAseq analysis (a) or qPCR (b); individual data points are shown with bar graphs representing mean value and standard deviation; two-way ANOVA, Tukey's test, $p$-value ${ }^{*}(<0.05),{ }^{* *}(<0.01),{ }^{* * *}(<0.001)$, $* * * *(<0.0001)$. 
bioRxiv preprint doi: $h t t p s: / / d o i . o r g / 10.1101 / 2021.03 .05 .433921$; this version posted March 13,2021 . The copyright holder for this preprint (which was not certified by peer review) is the author/funder, who has granted bioRxiv a license to display the preprint in perpetuity. It is made available under aCC-BY-NC-ND 4.0 International license.

Cambuli, Foletto, et al.

Supplementary Figure 11

a

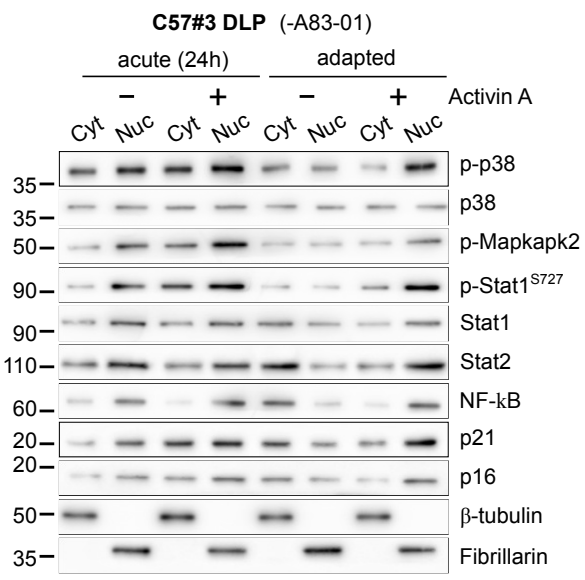

b

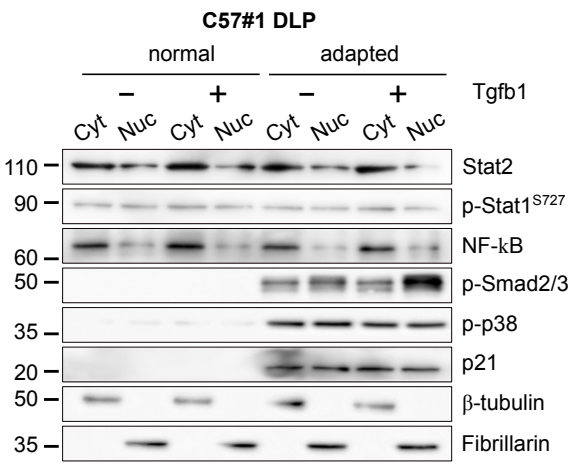

C

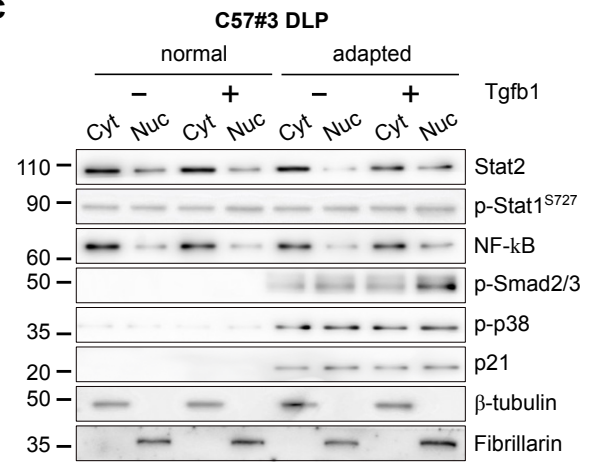

\section{Restoration of Activin A signalling - not Tgb1 - leads to cytostasis in organoid lines (C57 \#1 and \#3) adapted to grow in the absence of A83-01.}

a. Western blot analysis of C57\#3 DLP mouse prostate organoid line upon acute removal (24 hours) or adapted to grow without A83-01, in the presence or absence of Activin A (50 ng/mL, 24 hours).

b. Western blot analysis of C57\#1 DLP normal or adapted to grow without A83-01 mouse prostate organoid lines, in the presence or absence of Tgfb1 $(500 \mathrm{ng} / \mathrm{mL}, 24$ hours).

c. Western blot analysis of C57\#3 DLP normal or adapted to grow without A83-01 mouse prostate organoid lines, in the presence or absence of Tgfb1 (500 ng/mL, 24 hours). 


\section{Supplementary Table 1: Growth factors and small molecules}

\begin{tabular}{|l|l|c|}
\hline \multicolumn{1}{|c|}{ Treatment } & \multicolumn{1}{c|}{ Catalogue \# } & Concentration \\
\hline Activin A & R\&D, 338-AC & $50 \mathrm{ng} / \mathrm{mL}$ \\
\hline Tgfb1 & SinoBiol, 50698-M08H & $500 \mathrm{ng} / \mathrm{mL}$ \\
\hline Follistatin & PeproTech, 120-13 & $500 \mathrm{ng} / \mathrm{mL}$ \\
\hline SB203580 & Tocris, 1202 & $10 \mu \mathrm{M}$ \\
\hline SB202190 & PeproTech, 1523072 & $10 \mu \mathrm{M}$ \\
\hline SB431542 & Sigma Aldrich, S4317 & $10 \mu \mathrm{M}$ \\
\hline Ralimetinib* & Selleckchem, S1494 & $1 \mu \mathrm{M}$ \\
\hline Pirfenidone & Cayman, 13986 & $10 \mu \mathrm{M}$ \\
\hline Takinib & Sigma-Aldrich, SML2216 & $5 \mu \mathrm{M}$ \\
\hline IFN $\beta$ & SinoBiol, 50708-MCCH5 & $50 \mathrm{ng} / \mathrm{mL}$ \\
\hline
\end{tabular}

*Organoids were pre-treated with Ralimetinib $(1 \mu \mathrm{M})$ for 24 hours before being seeded for the experiments. 
bioRxiv preprint doi: $h t t p s: / / d o i . o r g / 10.1101 / 2021.03 .05 .433921$; this version posted March 13, 2021. The copyright holder for this preprint (which was not certified by peer review) is the author/funder, who has granted bioRxiv a license to display the preprint in perpetuity. It is made available under aCC-BY-NC-ND 4.0 International license.

Cambuli, Foletto, et al.,

Supplementary Table 2

Supplementary Table 2: Primary antibodies used for IF, IHC and flow cytometry

\begin{tabular}{|l|l|c|}
\hline Antigen & Catalogue \# & Dilution \\
\hline Krt5 & BioLegend, 905901 & $1: 500$ \\
\hline Krt8 & Merk, MAB329 & $1: 200$ \\
\hline Krt7 & Abcam, ab68459 & $1: 250$ \\
\hline Krt8/18 & Abcam, ab53280 & $1: 1000$ \\
\hline Ki67 & eBioscience, 14-5698-82 & $1: 200$ \\
\hline Egfr & Abcam, ab52894 & $1: 200$ \\
\hline Acvr1b & R\&D Systems, AF1477 & $1: 500$ \\
\hline Ppp1r1b & Invitrogen, MA5-14968 & $1: 400$ \\
\hline Ar & Santa Cruz Biotech, SC-816 & $1: 500$ \\
\hline Cd24a & eBioscience, BMS17-0242-82 & $1: 800$ \\
\hline Sca-1 & BioLegend, 122514 & $1: 800$ \\
\hline
\end{tabular}




\section{Supplementary Table 3: Primers used for end-point and quantitative PCR}

\begin{tabular}{|c|c|}
\hline Gene & Primers Sequence \\
\hline \multirow{2}{*}{ Ifi27 } & $f w: 5^{\prime}$-ggcttccattgtctccaaga-3' \\
\hline & $r v: 5^{\prime}$-accttcagtgctccaagtgc-3' \\
\hline \multirow{2}{*}{ Isg20 } & fw: $5^{\prime}$-cttctgaaaggcaagctggt-3' \\
\hline & $r v: 5^{\prime}$-tcttgtgtagcaggcgctta-3' \\
\hline \multirow{2}{*}{$\operatorname{Irf9}$} & fw: 5'-ctctttgttcagcgcctttg-3' \\
\hline & $r v-5$ '-tcccagaaatgtagggttgc-3' \\
\hline \multirow{2}{*}{ Isg15 } & $f w: 5^{\prime}$-gagctagagcctgcagcaat-3' \\
\hline & $r v: 5^{\prime}$-taagaccgtcctggagcact-3' \\
\hline \multirow{2}{*}{ Oasl2 } & fw: 5'-ctccggttggtgaagttctg-3' \\
\hline & $r v: 5^{\prime}$-gtcgcggtagtttacgagga-3' \\
\hline \multirow{2}{*}{$\operatorname{Irf7}$} & $f w: 5^{\prime}$-gaagaccaacttccgctgtg-3' \\
\hline & $r v: 5^{\prime}$-agcattgctgaggctcactt-3' \\
\hline \multirow{2}{*}{$\operatorname{Trp} 63$} & fw: 5'-agaacggcgatggtacgaag-3' \\
\hline & $r v: 5^{\prime}$-tctcacgacctctcactggt-3' \\
\hline \multirow{2}{*}{$A r$} & fw: 5'-gccaggagtggtgtgtgccg-3' \\
\hline & $r v:$ 5'-aagttgcggaagccaggcaagg-3' \\
\hline \multirow{2}{*}{ Tmprss 2} & $f w: 5^{\prime}$-gccgectccggagatttaag-3' \\
\hline & $r v: 5^{\prime}$-aggtgaccctgagttcaatgc-3' \\
\hline \multirow{2}{*}{ Psca } & $f w: 5^{\prime}$-gctcactgcaaccatgaaga-3' \\
\hline & $r v: 5^{\prime}$-gctaagtaggtggccagcag-3' \\
\hline \multirow{2}{*}{$N k x 3.1$} & $f w: 5^{\prime}$-cggaccctaggagggactc-3' \\
\hline & $r v: 5^{\prime}$-cacctgagtgtgagagaaggc-3' \\
\hline \multirow{2}{*}{ Mme } & $f w: 5^{\prime}$-ctccaacttctcccaatccc-3' \\
\hline & $r v: 5^{\prime}$-tcgagcagctgattttatgcagt-3' \\
\hline \multirow{2}{*}{$P b s n$} & fw: 5'-tgcacagtatgaagggagcat-3' \\
\hline & $r v: 5^{\prime}$-tccgtgtccatgatacgctg-3' \\
\hline \multirow{2}{*}{ Fkbp5 } & fw: 5'-agccgggaagcctaagtttg-3' \\
\hline & $r v-5$-acttgcctcccttgaagtaca-3' \\
\hline \multirow{2}{*}{$\operatorname{Tgm} 4$} & $f w: 5^{\prime}$-ctagagtcttgacagggctgc-3' \\
\hline & $r v-5$ '-tggtactcggatgtgtggtg-3' \\
\hline \multirow{2}{*}{$S b p$} & $f w: 5^{\prime}$-ccagattgtaccacaaaccctc-3' \\
\hline & $r v-5^{\prime}$-ccgtcctccagaaggaagtc-3' \\
\hline \multirow{2}{*}{ Sdha } & $f w: 5^{\prime}$-tggtgagaacaagaaggcatca-3' \\
\hline & $r v: 5^{\prime}$-cgcctacaaccacagcatca-3' \\
\hline \multirow{2}{*}{$T b p$} & $f w: 5^{\prime}$-cggtcgcgtcattttctccgc-3' \\
\hline & $r v: 5^{\prime}$-gtggggaggccaagccctga-3' \\
\hline \multirow{2}{*}{$A c t b$} & fw: 5'-atccagctcctagcacgcacg-3' \\
\hline & $r v: 5^{\prime}$-gtccccgctttgcgcaggtg-3' \\
\hline \multirow{2}{*}{ Gapdh } & fw: 5' -gagagtgtttcctcgtcccg-3' \\
\hline & $r v: 5^{\prime}$-actgtgccgttgaatttgcc-3' \\
\hline
\end{tabular}


bioRxiv preprint doi: https://doi.org/10.1101/2021.03.05.433921; this version posted March $13,2021$. The copyright holder for this preprint (which was not certified by peer review) is the author/funder, who has granted bioRxiv a license to display the preprint in perpetuity. It is made available under aCC-BY-NC-ND 4.0 International license.

Cambuli, Foletto, et al.,

Supplementary Table 4: Primary antibodies used for western blot

\begin{tabular}{|l|l|c|}
\hline Antigen & Catalogue \# & Dilution \\
\hline$\beta$-Actin & Merk, A2228 & $1: 2000$ \\
\hline -Tubulin & Santa Cruz Biotech, sc-5274 & $1: 4000$ \\
\hline Akt & CST, 9272 & $1: 2000$ \\
\hline Akt phosphoSer473 & CST, 4060 & $1: 1000$ \\
\hline Akt phosphoThr308 & CST, 9275 & $1: 1000$ \\
\hline Ar & Santa Cruz Biotech, sc-816 & $1: 500$ \\
\hline Atf2 anti-phosphoThr71 & CST, 9221 & $1: 1000$ \\
\hline Atm & CST, 2873 & $1: 1000$ \\
\hline Atm phosphoSer1981 & CST, 5883 & $1: 1000$ \\
\hline c-Myc & Abcam, ab32072 & $1: 1000$ \\
\hline Chk1 & CST, 2360 & $1: 1000$ \\
\hline Chk1 phosphoSer35 & CST, 2348 & $1: 1000$ \\
\hline Cyclin D1 & Abcam, ab134175 & $1: 1000$ \\
\hline Cyclin E1 & CST, 20808 & $1: 1000$ \\
\hline Erk & CST, 9102 & $1: 1000$ \\
\hline Erk phosphoThr202/Tyr204 & CST, 4370 & $1: 1000$ \\
\hline Fibrillarin & Abcam, ab4566 & $1: 500$ \\
\hline Gapdh & Thermo Fisher, MA515738 & $1: 4000$ \\
\hline Krt5 & BioLegend, 905901 & $1: 500$ \\
\hline Krt8/18 & Abcam, ab53280 & $1: 1000$ \\
\hline Mapkapk2 phosphoThr334 & CST, 3041 & $1: 1000$ \\
\hline p16 & Abcam, ab211542 & $1: 500$ \\
\hline p21 & Abcam, ab109520 & $1: 500$ \\
\hline p38 & CST, 9212 & $1: 1000$ \\
\hline p38 phosphoThr180/Tyr182 & CST, 9215 & $1: 500$ \\
\hline Smad2 phosphoSer465/467 & CST, 8828 & $1: 1000$ \\
\hline Smad2/3 & CST, 8685 & $1: 1000$ \\
\hline Smad4 & Santa Cruz Biotech, sc-7966 & $1: 1000$ \\
\hline Stat1 & BD Biosciences, 610186 & $1: 1000$ \\
\hline Stat1 phosphoSer727 & CST, 9177 & $1: 1000$ \\
\hline Stat1 phosphoTyr701 & CST, 9171 & $1: 1000$ \\
\hline Stat2 & CST, 4597 & $1: 1000$ \\
\hline Stat3 & CST, 9132 & $1: 1000$ \\
\hline Stat3 phosphoTyr705 & CST, 9131 & $1: 1000$ \\
\hline Stat5 & CST, 9358 & $1: 1000$ \\
\hline Stat5 phosphoTyr694 & CST, 9359 & CST, 5206 \\
\hline Tak1 & Abcam, ab26 & \\
\hline Trp53 & & 1000 \\
\hline
\end{tabular}

